\title{
Single-Cell Transcriptomics of Human Oocytes: Environment-Driven Metabolic Competition and Compensatory Mechanisms During Oocyte Maturation
}

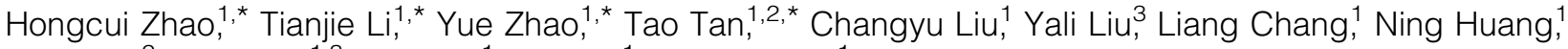
Chang Li,, Yong Fan, ${ }^{1,3}$ Yang Yu, Rong Li, and Jie Qiao ${ }^{1}$

\section{Abstract}

Aims: The mechanisms coordinating maturation with an environment-driven metabolic shift, a critical step in determining the developmental potential of human in vitro maturation (IVM) oocytes, remain to be elucidated. Here we explored the key genes regulating human oocyte maturation using single-cell RNA sequencing and illuminated the compensatory mechanism from a metabolic perspective by analyzing gene expression.

Results: Three key genes that encode CoA-related enzymes were screened from the RNA sequencing data. Two of them, ACATl and HADHA, were closely related to the regulation of substrate production in the Krebs cycle. Dysfunction of the Krebs cycle was induced by decreases in the activity of specific enzymes. Furthermore, the activator of these enzymes, the calcium concentration, was also decreased because of the failure of influx of exogenous calcium. Although release of endogenous calcium from the endoplasmic reticulum and mitochondria met the requirement for maturation, excessive release resulted in aneuploidy and developmental incompetence. High nicotinamide nucleotide transhydrogenase expression induced NADPH dehydrogenation to compensate for the NADH shortage resulting from the dysfunction of the Krebs cycle. Importantly, high $\mathrm{NADP}^{+}$levels activated DPYD to enhance the repair of DNA double-strand breaks to maintain euploidy.

Innovation: The present study shows for the first time that exposure to the in vitro environment can lead to the decline of energy metabolism in human oocytes during maturation but that a compensatory action maintains their developmental competence.

Conclusion: In vitro maturation of human oocytes is mediated through a cascade of competing and compensatory actions driven by genes encoding enzymes. Antioxid. Redox Signal. 30, 542-559.

Keywords: human oocytes, maturation, transcriptome sequencing, tricarboxylic acid cycle, NADPH/NADH

\footnotetext{
${ }^{1}$ Beijing Key Laboratory of Reproductive Endocrinology and Assisted Reproductive Technology and Key Laboratory of Assisted Reproduction, Ministry of Education, Center for Reproductive Medicine, Department of Obstetrics and Gynecology, Peking University Third Hospital, Beijing, China.

${ }^{2}$ Yunnan Key Laboratory of Primate Biomedical Research, Institute of Primate Translational Medicine, Kunming University of Science and Technology, Kunming, China.

${ }^{3}$ Key Laboratory for Major Obstetric Diseases of Guangdong Province, The Third Affiliated Hospital of Guangzhou Medical University, Guangzhou, China.

*These authors contributed equally to this work.

(C) Hongcui Zhao et al., 2018; Published by Mary Ann Liebert, Inc. This Open Access article is distributed under the terms of the Creative Commons Attribution Noncommercial License (http://creativecommons.org/licenses/by-nc/4.0), which permits any noncommercial use, distribution, and reproduction in any medium, provided the original authors and the source are cited.
} 


\section{Innovation}

Human oocytes are invaluable resources in clinical and basic research, but there is no information about the mRNA transcription changes during oocyte maturation. Recently, metabolism has been highlighted as a mediator that maintains its developmental potential during maturation. The present study shows for the first time that exposure to an in vitro environment can lead to dynamic changes in the global transcriptome in human oocytes, and it demonstrates that exposure to an in vitro environment can lead to dysfunction of the Krebs cycle in human oocytes during maturation; however, a compensatory action mediated by nicotinamide nucleotide transhydrogenase maintains their energy requirements, supporting developmental competence.

\section{Introduction}

$\mathbf{M}$ ATURATION IS A PREREQUisite for oocytes to accumulate enough energy and nutritional materials to support early-stage embryonic development before zygotic genomic activation. An in vitro maturation (IVM) system has been established successfully in rodents and domestic animals and even in humans to study this intrinsic mechanism (12). The IVM method is also used in the clinical setting as one component of assisted reproductive technologies (6).

The poor developmental potential of IVM oocytes has been noted in various species, particularly in humans, although $>5000$ human babies have been born via the IVM procedure worldwide (6), which has been attributed to an in vitro environment that can support the resumption of meiosis among immature oocytes. Failure of synchronization between the cytoplasmic and nuclear maturation leads to the poor acquisition of developmental competence by IVM oocytes (17). Factors that impair the resumption of meiosis and spindle assembly in IVM oocytes have been noted in previous studies using animal models and human materials (15, 46, 61 ), but the underlying molecular mechanism of cytoplasmic maturation driven by an in vitro environment is unknown, especially in human oocytes.

Metabolism plays an important role in regulating gene expression, protein translation, and protein modification. Insight into oocyte maturation has come from studies of specific metabolic pathways, including the pentose phosphate pathway, the Krebs cycle, and NADH catabolism (50). The normal function of these metabolic pathways provides necessary products for cellular physiology, such as the cell cycle, cytokinesis, and intracellular transport; however, dysfunction of these metabolic pathways results in apoptosis, autophagy, and failure of meiosis resumption $(11,57)$. In considering the differences between the in vitro and in vivo environments in which oocytes mature, it is necessary to investigate the dynamic changes in enzymes and their encoding genes under in vitro conditions.

Aberrant metabolism usually induces the occurrence of oxidative stress in oocytes, and it is therefore harmful to the subsequent developmental potential. In humans, oxidative stress has often been identified in the follicular fluid of aged women (34) or of women with polycystic ovary syndrome (PCOS) (36), and it is regarded as a risk factor to induce im- mature oocyte and decrease oocyte quality. Moreover, studies in mouse proved that antioxidant supplementation can effectively improve oocyte maturation and developmental potential in aged or PCOS models $(8,26)$. The molecular mechanism of the maturation failure in oocytes caused by oxidative stress has been addressed from the perspective of histone acetyltransferase $(60,66)$, DNA methylation $(37)$, and chromosome segregation errors (43), but the previous studies were focused on the effects of oxidative stress on oocytes in pathological or physiological environments, and studies that explored oxidative stress during oocyte maturation in vitro remain scarce.

In 2009, Tang et al. successfully completed mouse oocyte and embryo transcriptome sequencing using only single cells (54). Furthermore, Xue et al. analyzed the transcriptome in human oocytes and embryos using single-cell transcriptome sequencing technology and described the differences in genetic programs between human and mouse (58). Yan et al. also described the characteristics of human oocytes and embryos based on the transcriptome and analyzed not only mRNA but also long noncoding RNA (59). The expression profile of the RNA transcriptome in human oocytes during maturation remains unknown.

Here we investigated the transcriptome characteristics of human oocytes matured in vitro and in vivo to gain a transcriptome-level understanding of how oocytes mature and to illuminate the differences between human IVM and in vivo (IVO) matured oocytes at the transcript level. By analyzing the key genes and pathways that regulate the maturation of human oocytes from the perspective of metabolism, we elucidated the mechanisms that maintain the developmental potential of human IVM oocytes.

\section{Results}

\section{Single-cell transcriptome analysis in human oocytes}

Single-cell transcriptome sequencing was performed using human IVM and IVO oocytes (Supplementary Fig. S1A; Supplementary Data are available online at www.liebertpub.com/ ars). The immature oocytes at the GV stage generally matured and expelled their first polar body in $\sim 24-28 \mathrm{~h}$. In all, we generated six data sets for single-cell transcriptome sequences at a sequencing depth of 60 million mapped reads per single cell. This unbiased experimental approach allowed us to detect and characterize the expression patterns of over 16,000 proteincoding and noncoding RNAs. Correlation plots for the expression of RefSeq genes for individual oocytes from the IVM and IVO groups are shown in Supplementary Figure S1B and C.

To avoid effects from an individual's genetic factors, we first compared sequencing data from paired IVM and IVO oocytes from each woman. In the comparison groups, there were 8290,9168 , and 11,263 differentially expressed genes (DEGs), respectively (Fig. 1A). We then found the DEGs in common across the three comparison groups, which resulted in 2230 genes that displayed common differences between human IVM and IVO oocytes (Fig. 1B). Samples from the IVM and IVO groups were further distinguished by supervised hierarchical clustering and principal component analysis (Fig. 1C, D). These genes mainly consisted of protein-coding mRNAs (1523) and long noncoding RNAs (232) (Fig. 1E and Supplementary Table S1). The top six categories of biological process, cellular component, and molecular function of these DEGs are shown in Supplementary Figure S2. 

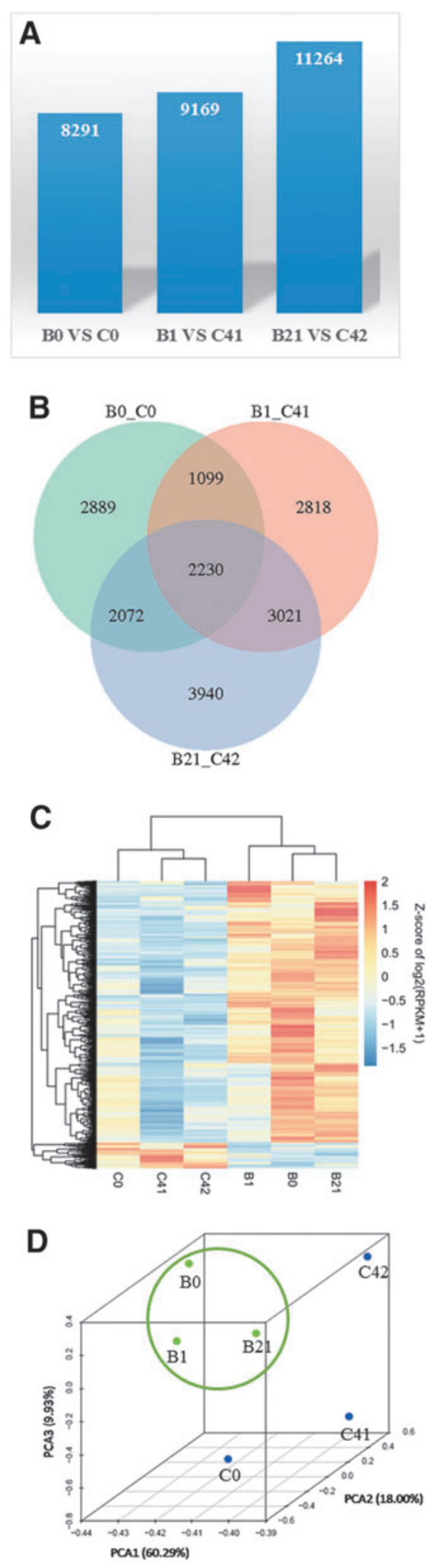

E

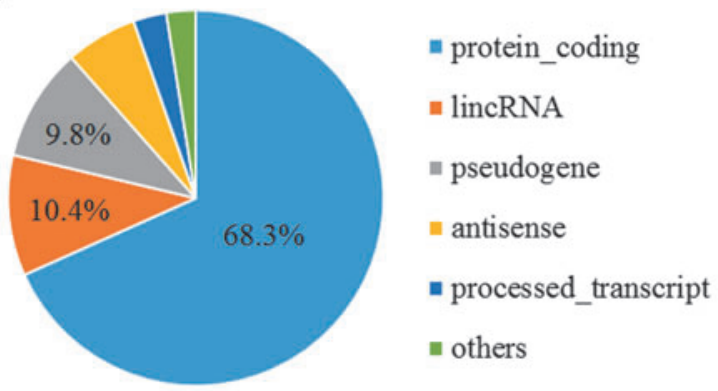

F

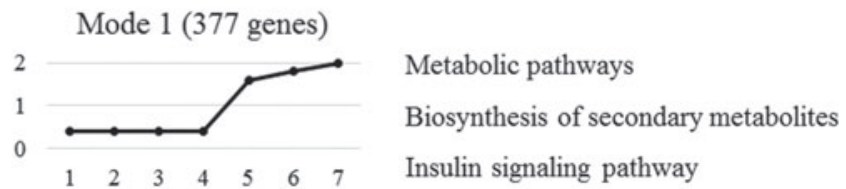

Mode 2 (269 genes)

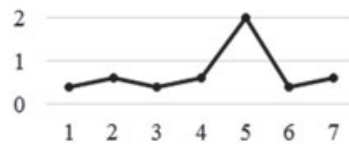

Metabolic pathways

PI3K-Akt signaling pathway

Purine metabolism

Mode 3 (213 genes)

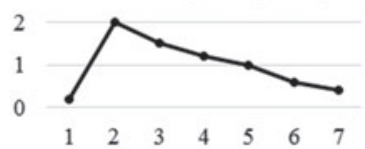

Metabolic pathways

MAPK signaling pathway

PI3K-Akt signaling pathway

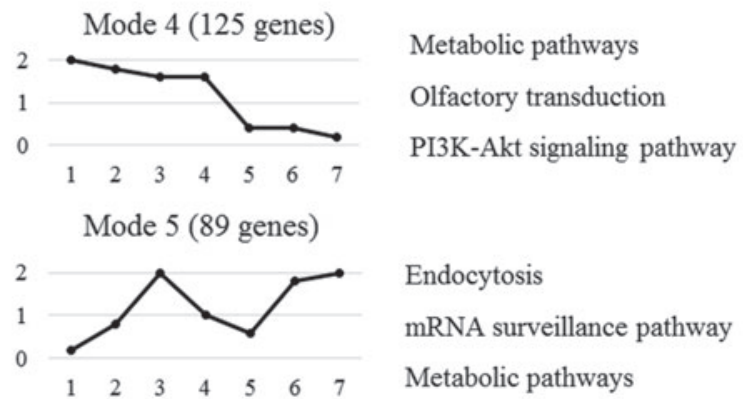

Mode 6 (77 genes)

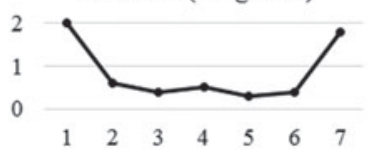

Metabolic pathways

PI3K-Akt signaling pathway

ECM-receptor interaction

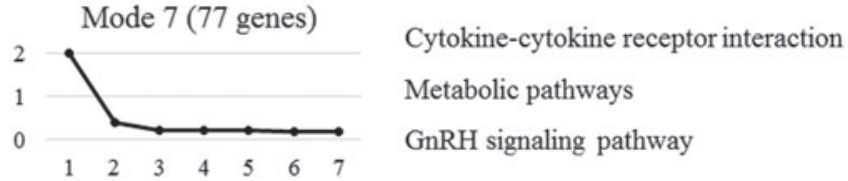

FIG. 1. Characteristics of and differences between transcriptomes from human IVM and IVO oocytes. (A) DEGs between IVM and IVO oocytes from single individuals. (B) Venn diagram showing the number of DEGs in common among the three sample pairs. (C) Human IVM and IVO oocytes can be clearly distinguished based on their transcriptome characteristics. The color key (from blue to red) of Z-score value (-1.5-2) indicates low to high expression levels. (D) Principal component analysis suggested that the three IVO oocytes clustered together, whereas the three IVM oocytes are distributed apart from the IVO oocytes. (E) In total, nine groups of genes were screened, with the majority consisting of protein-coding genes. (F) Representative expression profiles of DEGs during human embryo development. (1: MII oocytes, 2: zygotes, 3: two-cell stage, 4: four-cell stage, 5: eight-cell stage, 6: morulae, 7: blastocysts). (Group B was designated as IVM oocytes, and Group C was designated as IVO oocytes). DEG, differentially expressed gene; IVM, in vitro maturation; IVO, in vivo. 
The expression profiles of the 1523 protein-coding genes were checked for all early developmental stages from unfertilized oocytes to blastocysts using published data (59). Of these, 1461 genes could be categorized into 11 expression profiles (Supplementary Table S2). The seven most prevalent profiles were selected, and the three pathways that included the genes showing the greatest differential expression are shown in Figure $1 \mathrm{~F}$.

\section{Metabolic pathways and related genes were significantly impaired in IVM oocytes}

Pathways that could potentially be affected by IVM were analyzed. A total of 81 pathways were screened, and the top 10 pathways are shown in Supplementary Figure S3A. Among the pathways, $27 \%$ were related to metabolism, and $19 \%$ were signaling pathways (Supplementary Fig. S3B, Supplementary Tables S3 and S4).

To further screen the key genes regulating metabolism, we listed all of the genes in these 75 metabolic pathways, regardless of whether they were DEGs, in a sequencing database. Twenty-four genes that were involved in at least nine pathways were analyzed and were found to belong to the following six categories: the aldehyde dehydrogenase (ALDH) family, the mitogen-activated protein kinase (MAPK) family, the protein kinase (PRK) family, the phosphatidylinositol 3kinase (PI3K) family, the adenylyl cyclase (ADCY) family, and CoA-related enzymes (Supplementary Fig. S3C). Using the STRING database, the DEGs were clearly divided into two groups, one of which included the MAPK, PRK, PI3K, and ADCY families, and the other included the ALDH family and CoA-related enzymes (Supplementary Fig. S3D). The sixteen genes in the first four families encode kinases, and eleven of them displayed significant differences in expression between IVM and IVO oocytes (Supplementary Fig. S4). Among the other eight enzymes (ALDH family members and CoA-related enzymes), we found that four genes, encoding ACAT1, HADHA, DPYD, and ALDH2, showed the most significant differences (Supplementary Fig. S5).

To narrow down the number of important genes that are relevant to oocyte maturation, we collected high-quality (HQ) and low-quality (LQ) IVO oocytes based on our previously established criteria (64) and IVM oocytes (Fig. 2A). Three genes that encode CoA-related enzymes had the same expression profile in IVM oocytes as in LQ IVO oocytes, but their expression differed notably in HQ IVO oocytes (Fig. 2B).

The expression levels of the 24 genes in IVM and IVO oocytes were detected from different species, including mouse, rat, rabbit, and monkey. The results showed that gene expression profiles were more similar between human and monkey oocytes. There were four genes with consistent expression profiling in each group, including three genes encoding CoA-related enzymes and ALDH7A1 (Fig. 2C and Supplementary Table S5). Using qPCR, we found that in IVM oocytes compared with IVO oocytes, expression of $A C A T 1$ and $H A D H A$ was significantly decreased, whereas $D P Y D$ expression was significantly upregulated (Fig. 2D-F).

\section{Abnormal expression of ACAT1 and HADHA is a reflection of the impaired metabolic function in IVM oocytes}

Based on a bioinformatic analysis, we found that ACAT1 is involved in 16 metabolic pathways and $H A D H A$ is involved in 15 metabolic pathways. There are eight pathways in common between the two genes (Fig. 3A and Supplementary Table S6). Based on these pathways, we propose the following metabolic scenario (Fig. 3B). Deficient expression of ACAT1 and HADHA blocked the production of acetyl-CoA and succinate, which are the key substrates for the Krebs cycle, and therefore weakened the energy metabolism. Based on qPCR results, relative expression levels of 9 of the 15 genes encoding enzymes in the Krebs cycle were lower in the IVM oocytes than in the IVO oocytes (Fig. 3C). Immunofluorescence data showed that the ATP content of IVM oocytes was significantly lower than in the IVO group, however, mitochondrial content was not significantly decreased in the IVM group compared with IVO group. (Fig. 3D).

Mouse oocytes were used to study the effects of decreased expression of $A C A T 1$ and $H A D H A$ on maturation, fertilization, and subsequent development. First, we compared the expression levels of these 15 genes in mouse IVM and IVO oocytes (Supplementary Fig. S6A). Comparison of DEGs from human and mouse oocytes indicated that ten of these genes showed similar changes in expression (Supplementary Fig. S6B).

siRNAs for $A C A T 1$ and $H A D H A$ were coinjected into mouse GV oocytes, which resulted in effective knockdown of ACAT1 and HADHA expression (Supplementary Fig. S7A). Enzyme-linked immunosorbent assay (ELISA) results indicated that the activity of ACAT1, HADHA, OGDH, IDH3A, and SDHA was significantly decreased in siRNA oocytes relative to controls (Supplementary Fig. S7B). The gene expression results were similar to the results from comparing mouse and human IVM oocytes with their IVO controls (Supplementary Fig. S7C). Moreover, Immunostaining results suggested that oxidative stress as represented by ROS was significantly increased in both the cytoplasm and mitochondria, but the GSH level decreased in the siRNA group relative to the controls. Furthermore, the level of oxidized FAD $\left(\mathrm{FAD}^{++}\right)$was significantly enhanced, whereas the level of reduced NADH was significantly decreased in the siRNA injection group compared with the other two control groups (Supplementary Fig. S7D-G).

The maturation ratio was not impaired in the siRNA group (Supplementary Fig. S8A). siRNA and control oocytes were then fertilized by intracytoplasmic sperm injection (ICSI). The blastocyst formation and implantation rate was not affected by the injection with $A C A T 1$ and $H A D H A$ siRNA (Supplementary Fig. S8B, C), but the birth rate was significantly decreased (Supplementary Fig. S8D). ACAT1 and HADHA siRNA treatment led to a significant delay in the hatching of developing blastocysts, a decrease in the number of cells per blastocyst, and a higher incidence of DNA damage as indicated by the frequency of apoptotic cells (Supplementary Fig. 9A-E).

\section{Failure of the calcium signaling pathway accounts for the reduced activity of enzymes}

Calcium often acts as an activator for enzymes and is generally controlled via the ER or mitochondria (Fig. 4A). By analyzing the transcriptome data, we found that the calcium signaling pathway in IVM oocytes was potentially dysfunctional (Supplementary Fig. S10A). A supervised clustering result indicated that IVM and IVO oocytes can be clearly separated based on the expression of the 52 genes involved in this pathway (Supplementary Fig. S10B). Moreover, the average intensity of 

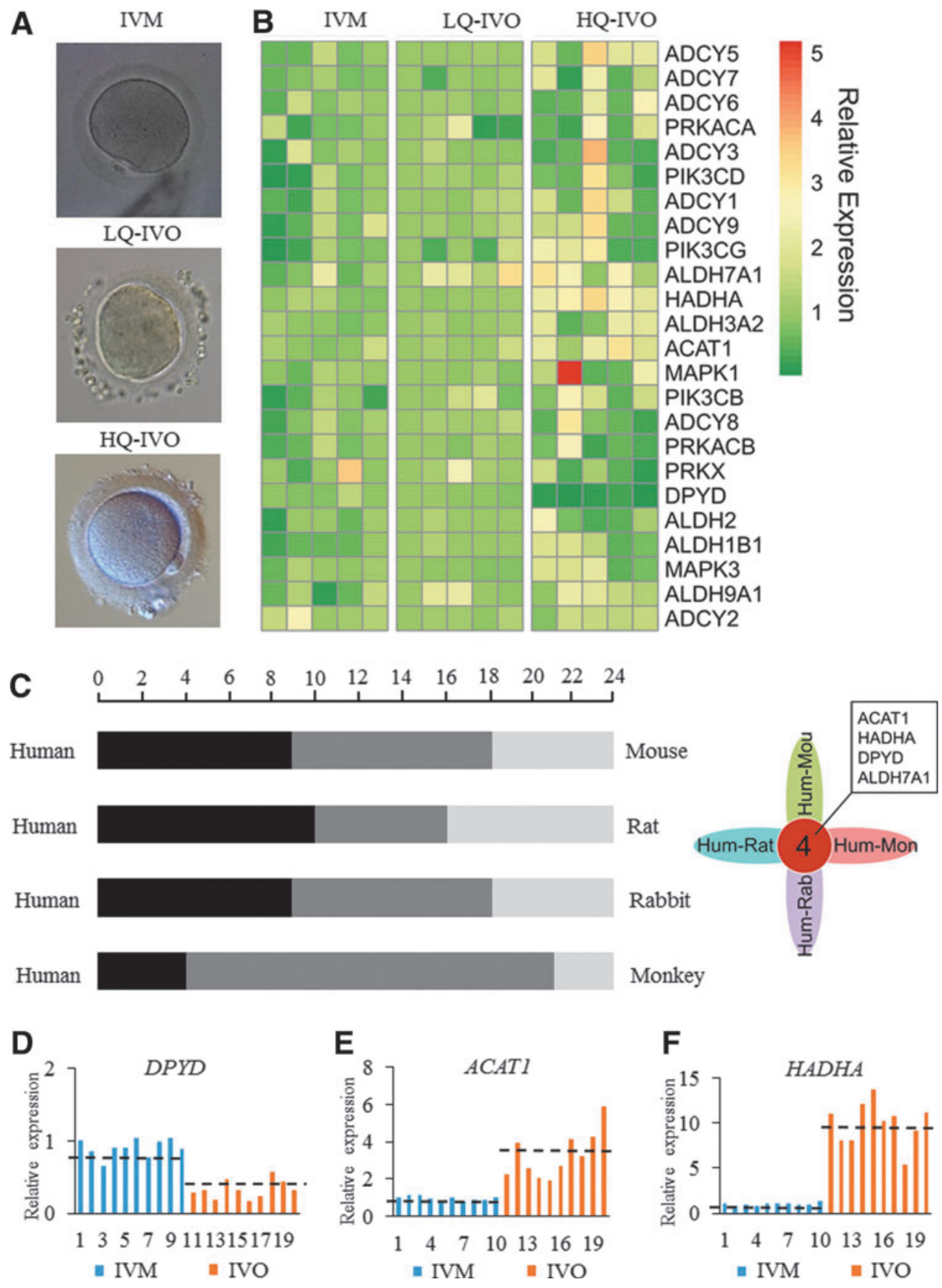

the calcium signal was also decreased in human IVM oocytes compared with human IVO oocytes (Fig. 4B). Genes encoding plasma membrane proteins that are related to calcium transport were all downregulated; however, genes encoding endoplasmic reticulum (ER) and mitochondrial membrane proteins were all upregulated (Fig. 4C, D). Moreover, genes encoding calmodulin (CAM) and $\mathrm{Ca}^{2+} /$ calmodulin-dependent protein kinase II (CAMKII) showed different expression profiles between IVM and IVO oocytes (Fig. 4E). These differences were

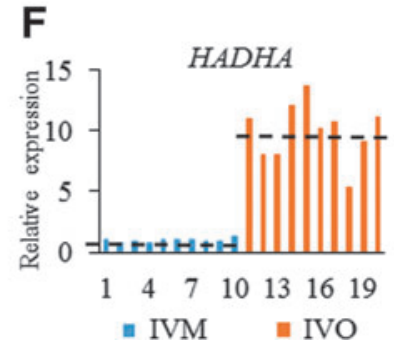

maintained at the protein level, as well as for PKA, based on immunostaining and dot blot analysis (Fig. 4F-K).

To confirm the effects of calcium concentration on human oocyte development, the human IVO oocytes were fertilized, and then, the fertilized embryos were cultured in a calciumfree medium. The fertilized embryos developed to the blastocyst stage with lower efficiency, and the derivation efficiency of embryonic stem (ES) cells was also decreased compared with normal controls (Supplementary Fig. S11A,

FIG. 3. Relationship of regulatory pathways for ACAT1 and HADHA. (A) Eight pathways were shared between ACAT1 and HADHA. (B) Schematic diagram of the proposed metabolic relationship between these two genes and the Krebs cycle. (C) Relative mRNA expression levels of genes encoding enzymes in the Krebs cycle in both IVM and IVO oocytes. (D) Identification of relative ATP (green color) and mitochondrial (red color) contents in both IVM and IVO oocytes. Bar $=100 \mu \mathrm{m}$, and $* p<0.05$. 

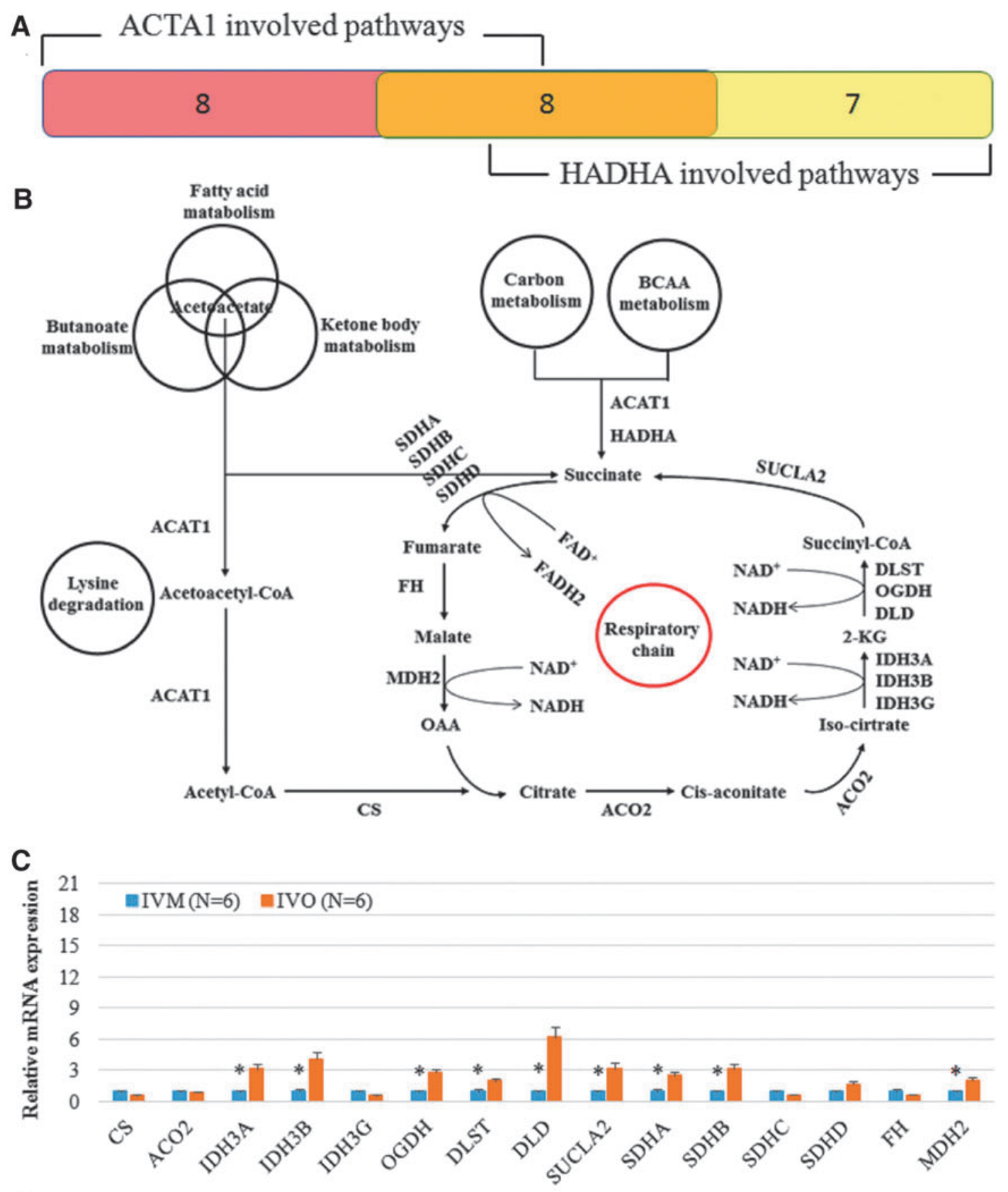

\section{D}
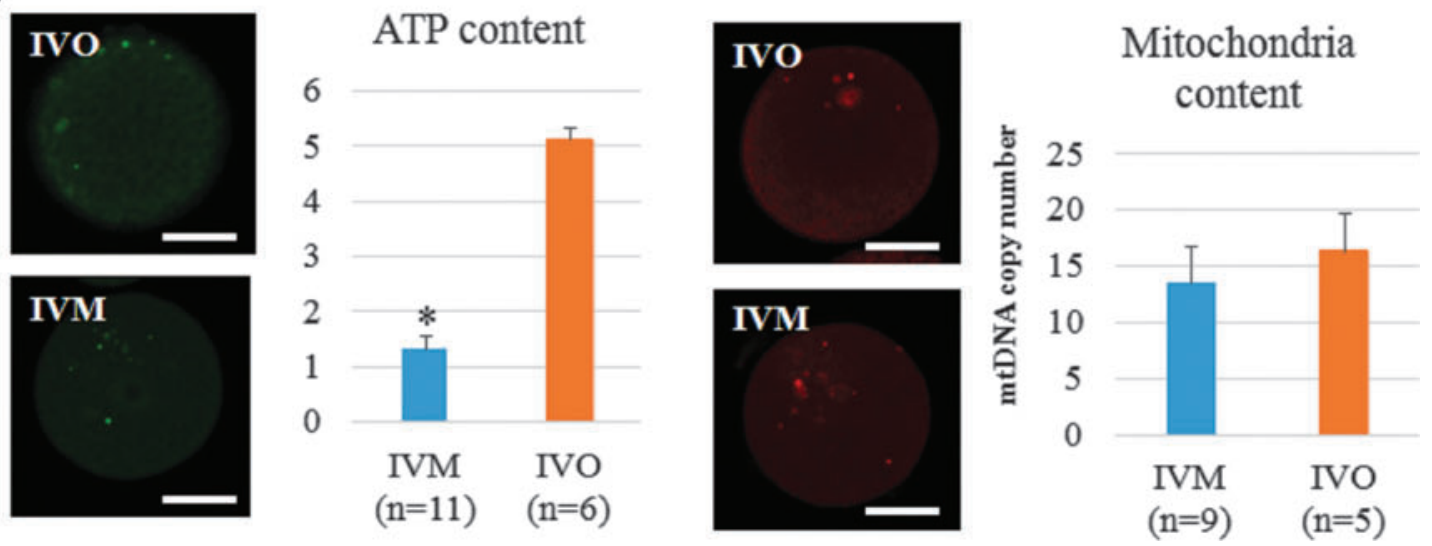


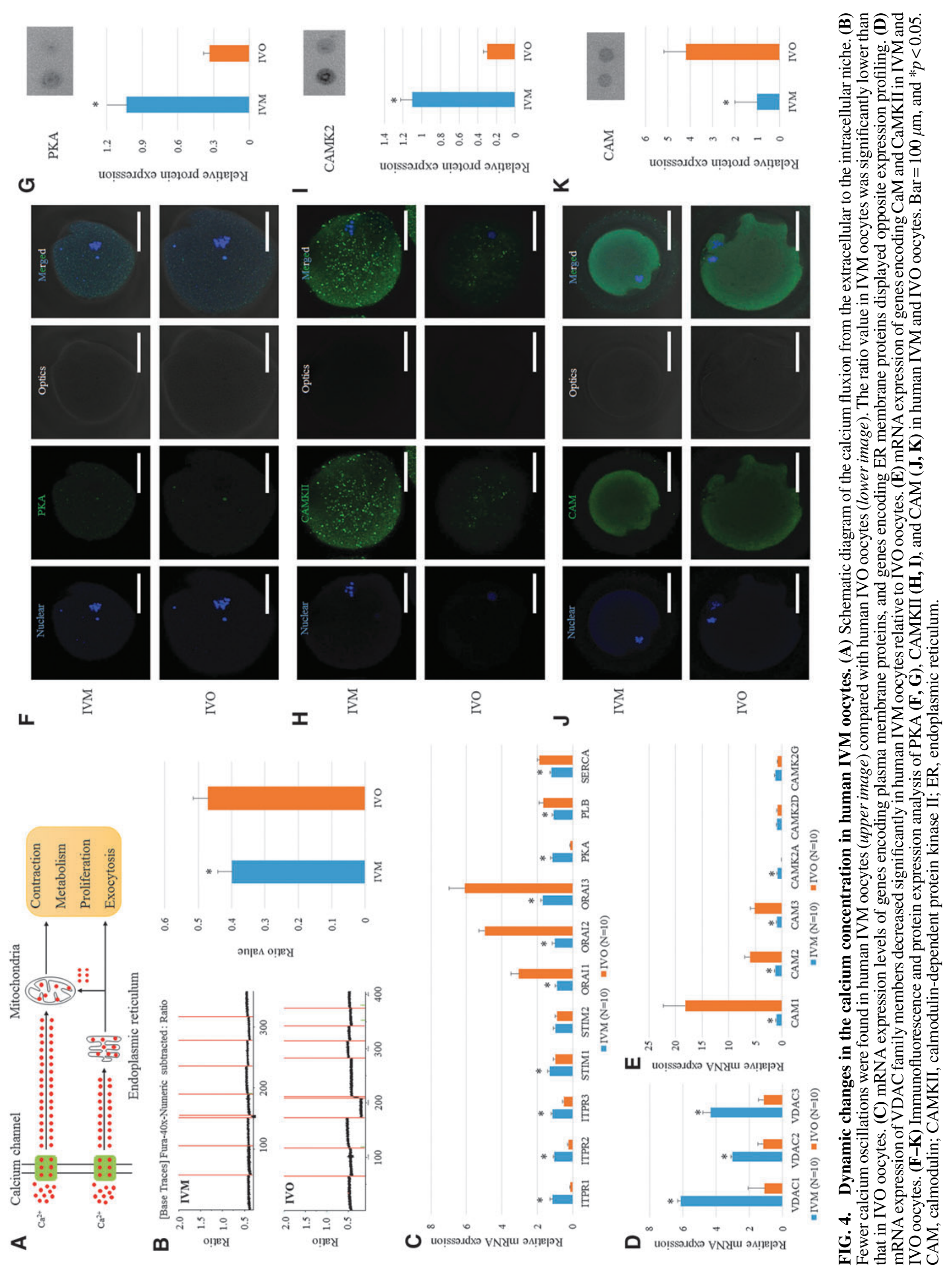


B). Moreover, these embryos showed microduplications or microdeletions in multiple chromosomes in trophectoderm (TE) cells of the resulting blastocysts (Supplementary Fig. S11C, D), and gene expression results indicated that energy metabolism was impaired (Supplementary Fig. S11E, F).

\section{Compensatory production of $\mathrm{NADH}$ and $\mathrm{NADP^{+ }}$ provides a developmental opportunity for IVM oocytes}

NADH is mainly produced by glycolysis and the Krebs cycle; however, glycolysis does not occur in oocytes, and the Krebs cycle is inactive during maturation in vitro. A potential compensatory mechanism is that NADPH transfers a hydrogen ion to $\mathrm{NAD}^{+}$and therefore generates $\mathrm{NADP}^{+}$and NADH (Fig. 5A). Encoding mRNA of NADPH-dependent antioxidants in our sequencing data was shown in Figure 5B, and the expression levels of $G P X$ and $P R D X$ family members have no notable changes. We analyzed the expression of the enzyme that catalyzes this reaction, nicotinamide nucleotide transhydrogenase (NNT). Gene and protein expression levels of NNT were both upregulated in IVM oocytes (Fig. 5C, D). Moreover, inhibition of NNT expression by siRNA in IVM oocytes resulted in developmental arrest at the eight-cell stage and significantly decreased blastocyst efficiency (Fig. 5E, F). We examined the mRNA and protein expression of NNT in multiple mouse IVM and IVO oocytes to confirm this difference (Supplementary Fig. S12A, B). When NNT expression was blocked by siRNA in mouse oocytes (Supplementary Fig. S13C), IVM oocytes were arrested at the two-cell stage more frequently than in the controls (Supplementary Fig. S12D). Although a few of the IVM embryos formed blastocysts, the quality of the blastocysts was still poor (Supplementary Fig. S12E). Immunostaining indicated that the levels of ROS in the cytoplasm and mitochondria (indicated by MitoSOX) and oxidized FAD were significantly increased, and that of GSH and NADH were significantly decreased in IVM oocytes with NNT-siRNA injection, compared with control samples (Supplementary Fig. S13A-D).

$\gamma \mathrm{H} 2 \mathrm{AX}$-positive signal, which reflects DNA double-strand breaks (DSBs) in chromosomes, was higher in IVM oocytes than in IVO oocytes (Fig. 6A, B), indicating the genome instability induced by the IVM process. With higher expression of NNT in human IVM oocytes, NADP ${ }^{+}$production was increased, which was consistent with the upregulated expression of DPYD, an $\mathrm{NADP}^{+}$-dependent enzyme that specifically repairs DSBs. In vivo, DPYD was highly expressed in the primary and secondary follicle but was not expressed in oocytes and preimplantation embryos (Fig. 6C); however, expression of DPYD increased in primary and secondary follicles incubated in vitro and was dramatically upregulated in IVM oocytes (Fig. 6D-F). Immunostaining results showed that the DPYD protein was often distributed around the cytomembrane in oocytes at the MII stage but was also localized to the GV in oocytes at the GV stage (Fig. 6G).

We then treated the oocytes with $100 \mu M \mathrm{H}_{2} \mathrm{O}_{2}$ to induce DSBs and found that the proportion of embryos with a $\gamma \mathrm{H} 2 \mathrm{AX}$-positive signal was significantly increased. These embryos were still able to develop to the blastocyst stage, although with lower efficiency, and DPYD expression was significantly upregulated (Supplementary Fig. S14A-C). After knocking down $D P Y D$ using $D P Y D$-specific siRNA, the embryos derived from $\mathrm{H}_{2} \mathrm{O}_{2}$-treated oocytes showed a high frequency of aneuploidy, and fewer of these embryos were able to form normal blastocysts (Supplementary Fig. S14DF). Representative images of developed embryos from the three groups are shown in Supplementary Figure S14G.

\section{Discussion}

In the present study, we analyzed the dynamic changes in global gene expression in human IVM and IVO oocytes. Single-cell sequencing with a next-generation sequencing platform has been widely applied in the fields of genetics (25), epigenetics (20), and RNA transcriptome $(58,59)$ analysis to establish relevant databases and explore the mechanism of development and differentiation. For human oocytes and fertilized embryos, there have been only two RNA sequencing studies because of the preciousness of human oocytes $(58,59)$. Xue et al. identified a sequence of transcriptional changes in multiple developmental pathways and suggested conserved key members (or hub genes) in the human and mouse networks (58). Subsequently, Yan et al. discovered 2733 novel long noncoding RNAs in human oocytes and embryos, providing a more comprehensive framework of the transcriptome landscape of human early embryos (59). The present study reveals the dynamic changes in the transcriptome during human oocyte maturation in vitro and therefore provides a series of molecular targets to improve the developmental outcome of human IVM oocytes.

Our study revealed that two groups of genes, those encoding kinases and those encoding enzymes related to CoA production, had significantly different expression levels between IVM and IVO oocytes. Kinases have an important role in activating the expression of key molecules during meiosis resumption (42), and CoA, in conjunction with enzymes, can catalyze reactions in the cytoplasm (56). Therefore, these two groups of genes represent the key regulators for nuclear and cytoplasmic maturation, which is recognized as fundamental for oocyte maturation. Although previous studies of nuclear maturation have analyzed changes in single genes or single gene families, ours is the first global analysis of these changes.

Cross talk between the nucleus and cytoplasm determines the eventual development outcome of IVM oocytes (1). Most previous studies focused on nuclear maturation and screened for the key molecules and pathways involved (48, 62, 65); however, resulting increases in the efficiency of IVM oocytes were negligible. Recently, more studies have focused on cytoplasmic maturation $(24,33,52)$. Organelles in oocytes undergo changes in biogenesis, morphology, and cellular pathways in IVM oocytes that lead to cellular stress and apoptosis, and these factors eventually contribute to the failure of cytoplasmic maturation. These results were confirmed in our study in the point view of global transcriptome.

Three CoA-related enzymes encoding genes were isolated in our analysis, ACAT1, HADHA, and DPYD. ACAT1 and $H A D H A$ are mainly involved in metabolic processes and contribute to the Krebs cycle $(13,35)$, which is impaired in IVM oocytes. ACAT1 encodes a mitochondrial enzyme that catalyzes the reversible formation of acetoacetyl-CoA from two molecules of acetyl-CoA (27). Transcription of ACAT1 is promoted by leptin (23), angiotensin II (28), and insulin (18) in human monocytes/macrophages. As mitochondrial acetoacetyl-CoA thiolase is involved in $\beta$-oxidation, deficiency in this enzyme is marked by increased levels of 
A Pathways to produce $\mathrm{NADH}$ in oocyte

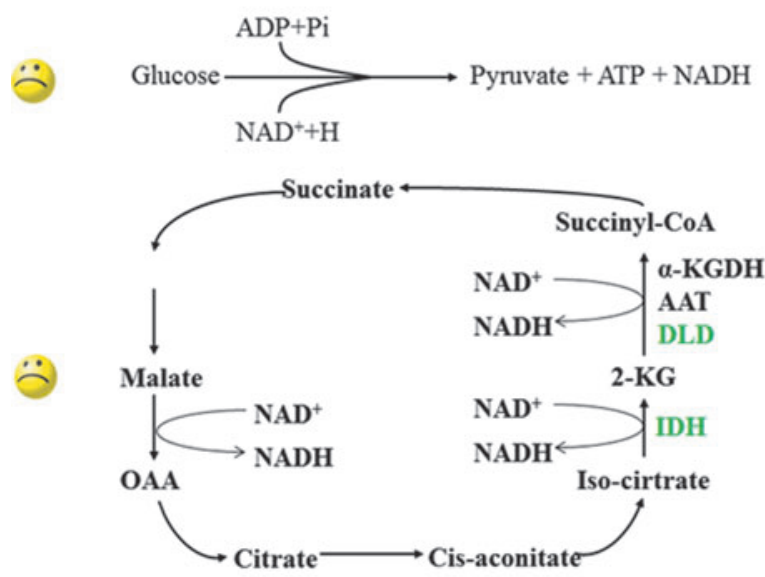

? $\mathrm{NADPH}+\mathrm{NAD}^{+} \longrightarrow \mathrm{NADP}^{+}+\mathrm{NADH}$
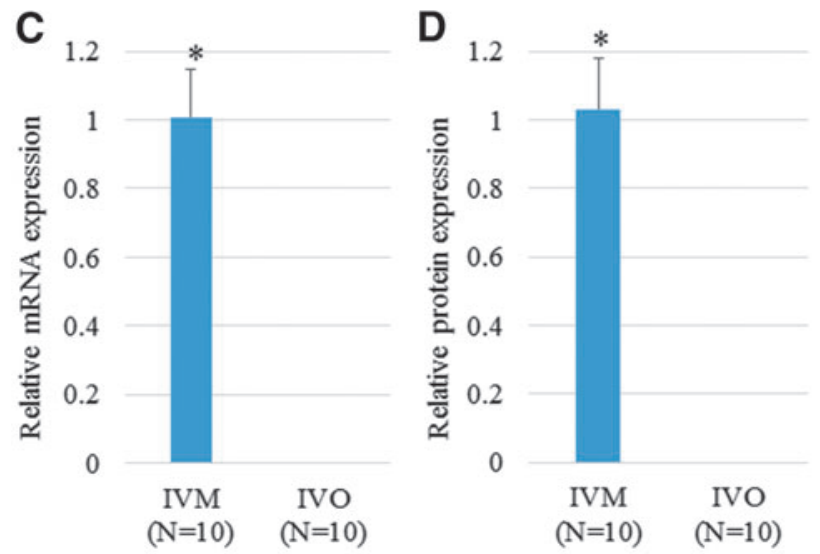

B
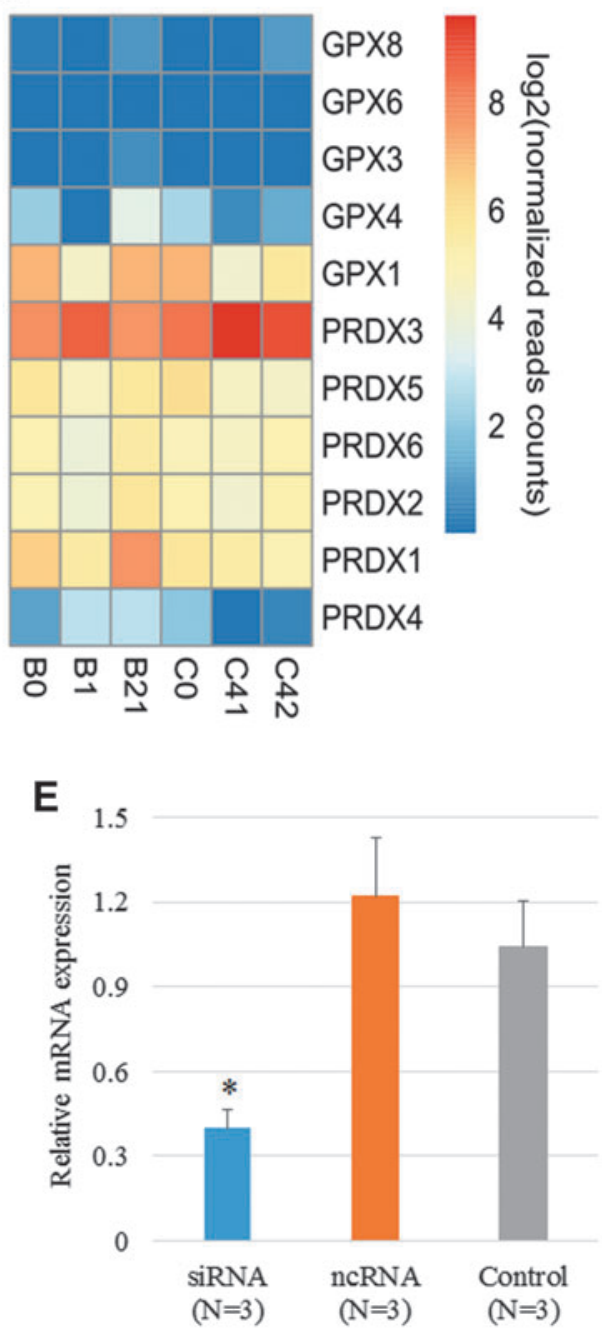

F 100

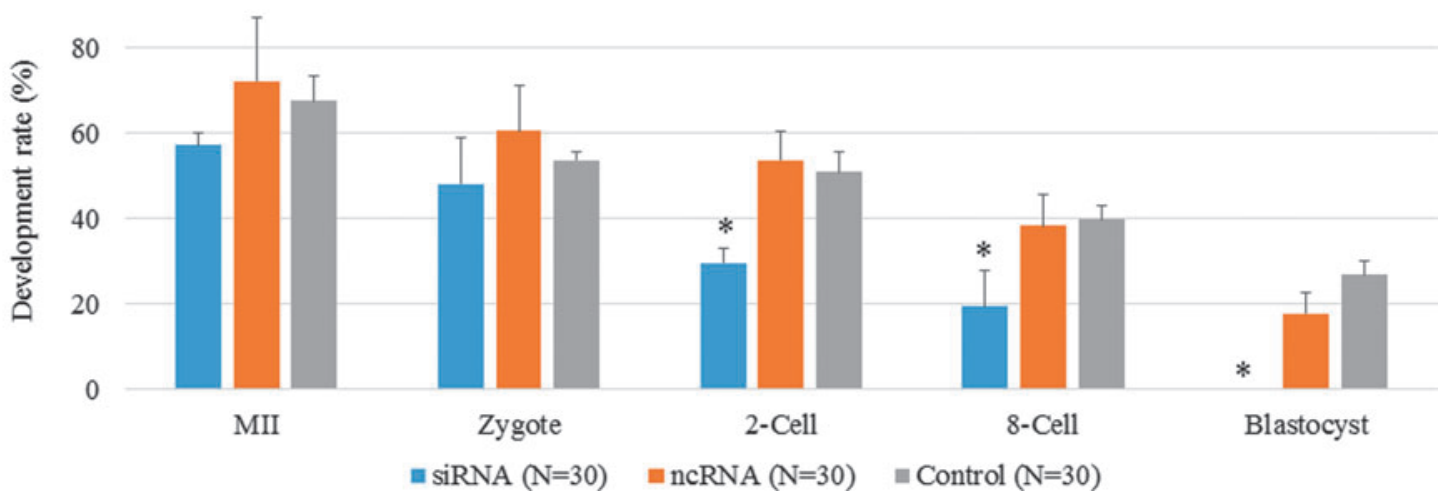

FIG. 5. Important roles of NNT in regulating the developmental competence of IVM oocytes. (A) Three pathways in which NADH was produced. (B) Expression profiles of GPX and PRDX family members that were NADPH-dependent antioxidants. (C, D) mRNA and protein expression of NNT was significantly higher in human IVM oocytes than in IVO oocytes. (E) NNT mRNA expression was inhibited by injection of NNT-specific siRNA into human oocytes. (F) Impaired preimplantation development of human oocytes after injection of $N N T$-specific siRNA. $* p<0.05$. NNT, nicotinamide nucleotide transhydrogenase. 
A

FIG. 6. In vitro culture induced the activation of DPYD in human germ cells at different stages. (A) Analysis of the $\gamma \mathrm{H} 2 \mathrm{AX}$ signal in human IVM and IVO oocytes. (B) The percentage of $\gamma \mathrm{H} 2 \mathrm{AX}$-positive embryos was significantly increased in human IVM oocytes. (C) DPYD mRNA expression during all stages of human female germ cells and in fertilized embryos. (D-F) In vitro culture significantly induced $D P Y D$ expression in primary follicles (D), secondary follicles $(\mathbf{E})$, and $\mathrm{MII}$ oocytes (F). (G) DPYD protein localization in human GV and MII oocytes. Bar $=50 \mu \mathrm{m}$. $* p<0.05$.

C

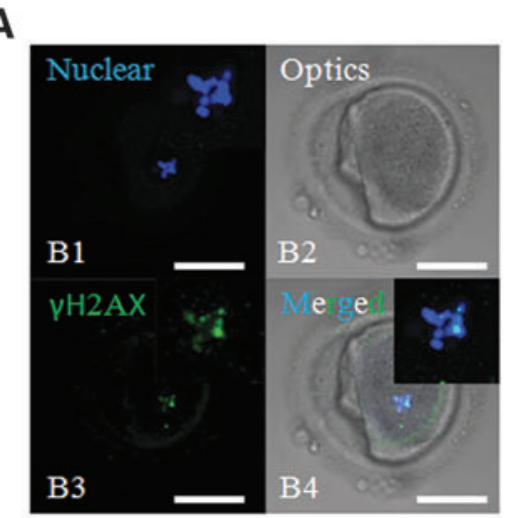

B
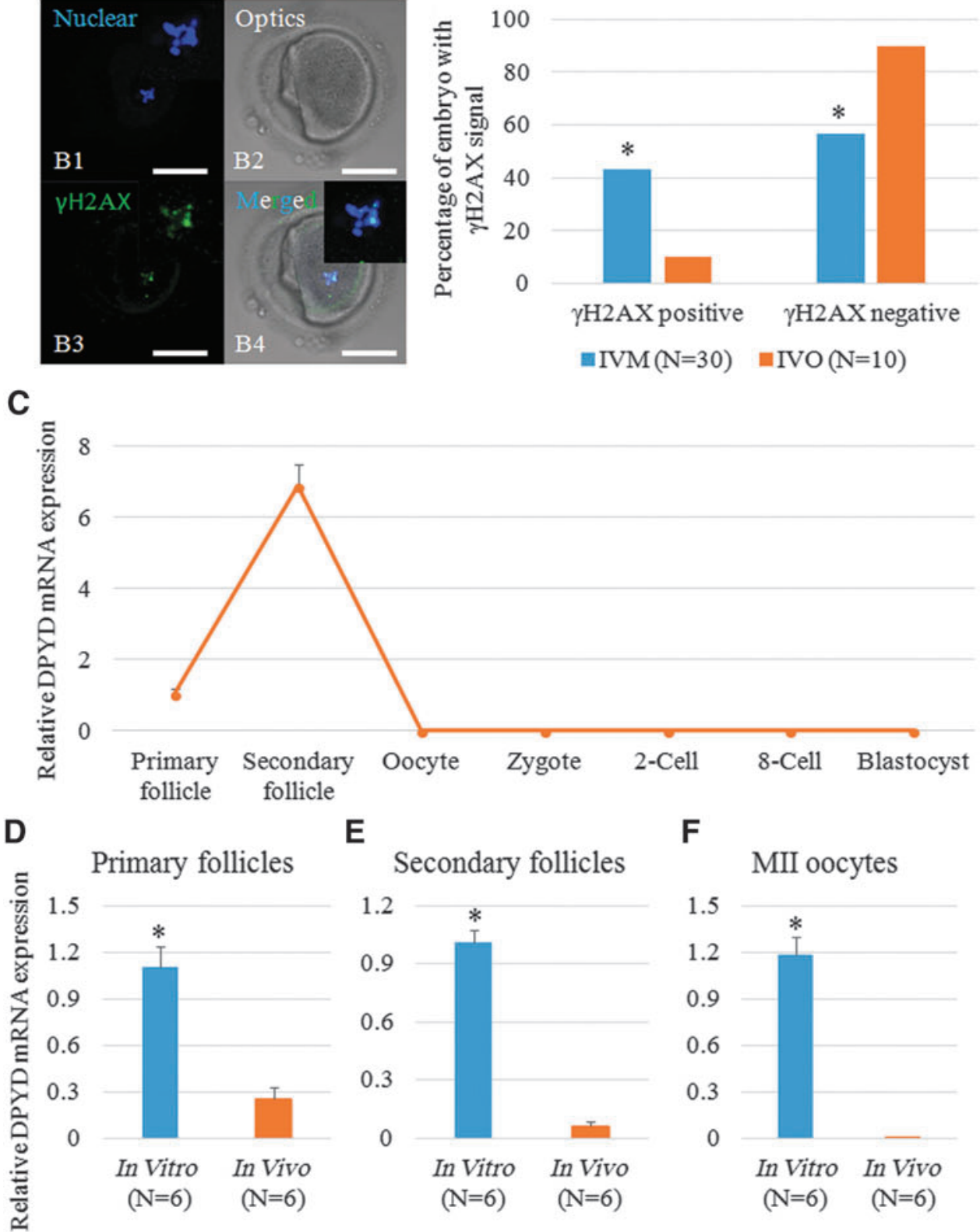

E

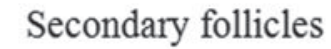

$\mathbf{F}$
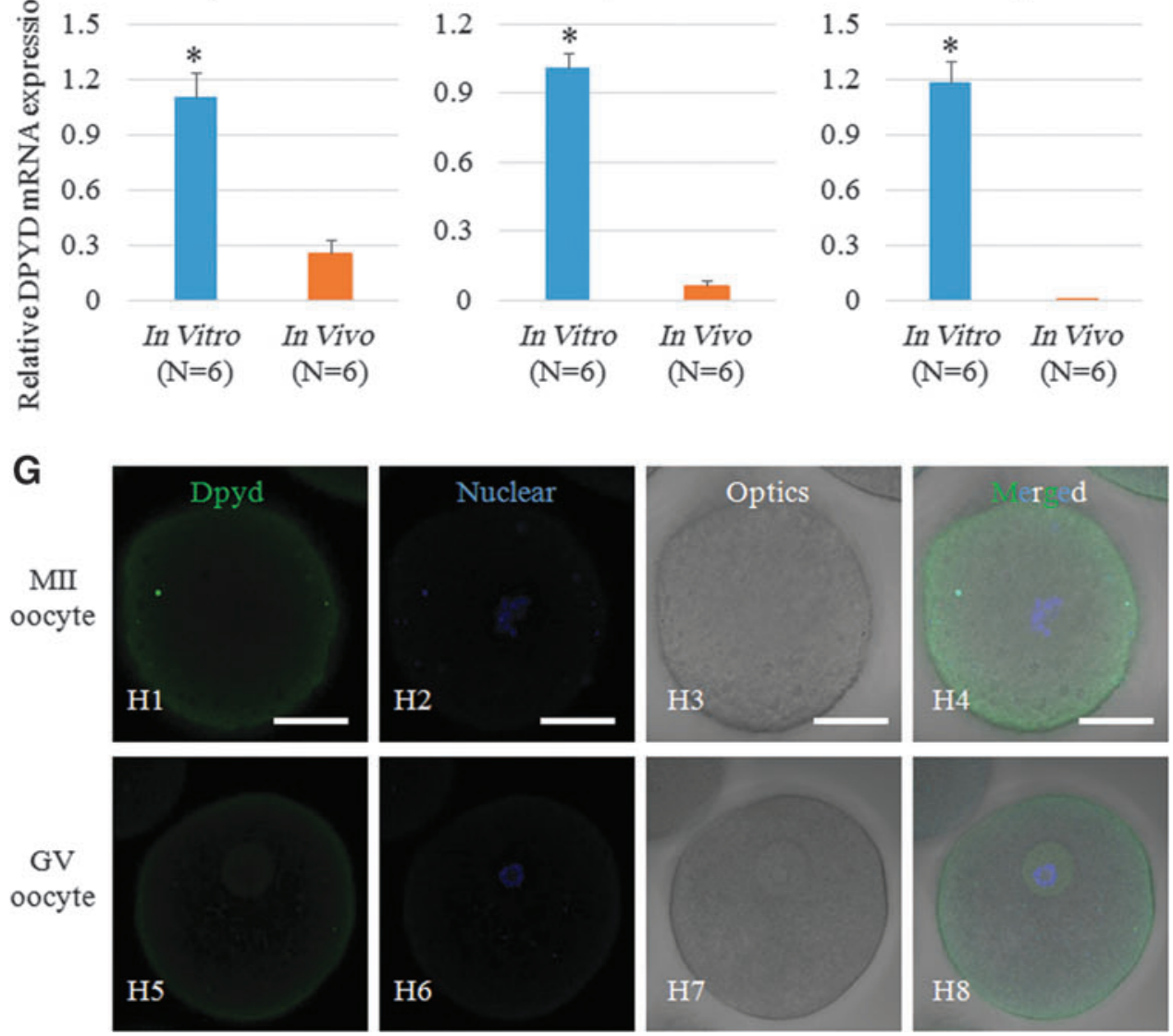
cholesterol compounds $(18,23,28)$. In addition, the isoleucine pathway is affected, such that proper metabolism of this amino acid is halted (29). González-Serrano et al. found that blastocysts produced in vitro and their in vivo counterparts differed significantly in the homeostasis of cholesterol and free fatty acid metabolism (19), suggesting that embryonic lipid metabolism is affected by in vitro incubation, and ACAT1 is a marker that reflects upstream lipid metabolism.

$H A D H A$ encodes the mitochondrial protein trifunctional enzyme subunit alpha. HADHA catalyzes the last three steps of mitochondrial $\beta$-oxidation of long-chain fatty acids (67). While NAD alone is present, HADHA can convert mediumand long-chain 2-enoyl-CoA compounds into 3-ketoacyl$\mathrm{CoA}$, whereas it results in the production of acetyl-CoA when NAD and CoA-SH are present (4).

A common characteristic of ACAT1 and HADHA is that both are mitochondrial and both are involved in mitochondrial $\beta$-oxidation. In mouse, bovine, and porcine oocytes, $\beta$ oxidation is involved in the regulation of meiosis resumption (41). In those studies, however, the cumulus-oocyte complex was often used, and it was therefore difficult to illuminate the importance of $\beta$-oxidation in the ooplasm rather than in the oocyte itself during maturation (41). Dunning et al. found that $\beta$-oxidation can be detected in denuded mouse oocytes, which indicates a potential role for $\beta$-oxidation in the ooplasm (10). In our study, we first indicated a potential relationship between $\beta$-oxidation and human oocyte maturation by finding two related genes, ACAT1 and HADHA.

In mouse, the mechanism by which impaired $\beta$-oxidation blocks maturation was mainly attributed to the effect on energy metabolism via the Krebs cycle and the electron transport chain. In our study, we also observed abnormal expression of genes involved in the Krebs cycle. Nine of a total of fifteen genes encoding ten enzymes or enzyme complexes in TCA showed abnormal expression profiling, including almost all enzymes that control NADH and FADH2 production.

There are three IDH isozymes in humans. IDH3 catalyzes the third step of the Krebs cycle while converting $\mathrm{NAD}^{+}$to $\mathrm{NADH}$ in the mitochondria, whereas IDH1 and IDH2 catalyze the same reaction beyond the context of the Krebs cycle and use $\mathrm{NADP}^{+}$as a cofactor instead of $\mathrm{NAD}^{+}(21)$. SuttonMcDowall et al. observed that IDH is a potential substrate for BMP15 and FSH, both of which can improve oocyte maturation (51). SDH is an enzyme complex that is bound to the inner mitochondrial membrane of mammalian mitochondria and to many bacterial cells. It is the only enzyme that participates in both the Krebs cycle and the electron transport chain (40). Boerjan et al. suggested that SDH activity is correlated with the location of mitochondria in preovulatory mouse oocytes, and its mitochondrial distribution is closely related to chromatin organization. Therefore, there is a potential correlation between SDH activity and chromatin morphology (2). The SDH complex has four isoforms, SDHA, SDHB, SDHC, and SDHD. In our results, we found that the expression of these four genes was dynamic and changed stochastically, with more than two isoforms being affected in human IVM oocytes. Regardless of which isoform is affected, when one isoform is mutated, the overall SDH complex becomes unstable and therefore loses its function, leading to reduced function of the Krebs cycle and electron respiration chain (31). That is, the overall dysfunction of enzymes resulted in reduced maturation and development of human IVM oocytes.
We then noticed that calcium is a common activator of these enzymes (5). From the beginning of fertilization, dynamic changes in the calcium concentration have a key role in promoting development (47). Impaired calcium homeostasis leads to the failure of oocyte maturation, fertilization, and development (22). Calcium in the cytoplasm is derived from two sources: one is intake from the extracellular environment, and the other is release from intracellular stores in the ER and mitochondria. Our study indicated that almost all genes encoding membrane proteins in the calcium metabolic pathway were downregulated, which indicates a potential intake barrier; however, intracellular calcium can still be detected in IVM oocytes, which may be attributable to an excessive release of calcium from the ER and mitochondria to complement this insufficient intake. Excessive calcium release would result in ER stress and therefore induce apoptosis and autophagy (30). In our study, genes involved in ER stress pathways were not stably expressed (data not shown), which suggests that the ER is involved in both releasing calcium and maintaining the calcium concentration in the cytoplasm, thereby inducing apoptosis and blocking development because of stress. Thus, inhibiting the ER stress response would improve the maturation and development of human IVM oocytes. In our study, we demonstrated that treating oocytes and embryos in in vitro culture with a lower calcium concentration would decrease the potential for embryonic development and increase the incidence of aneuploidy. The results again show the importance of calcium for regulating oocyte maturation and embryonic development.

Dysfunction of the Krebs cycle would impair NADH and ATP production, thereby compromising intracellular metabolism and mRNA transcription $(3,7)$. NADH, the reduced form of $\mathrm{NAD}^{+}$that is generated during cellular metabolism, is used to transfer the reductive potential captured from catabolic reactions into $\mathrm{NADH}$ ubiquinone oxidoreductase (complex I), which generates the membrane potential for ATP regeneration (44). In human IVM oocytes, we found that the two main pathways to produce NADH were weakened (deficient ability to carry out glycolysis (49) and an impaired Krebs cycle), but the activity of mitochondrial NNT was enhanced, which should facilitate the transfer of electrons between NADPH and NADH. Nickel et al. suggested that transformation from NADH to NADPH is a forward mode, but transformation from NADPH to NADH is a reverse mode and often induces a pathological outcome (39). In our study, we found that the NADPH concentration was decreased, which indicated a potential hydrogen loss and transformation from NADPH to NAD ${ }^{+}$. Human IVM oocytes also showed a decrease in developmental competence when NNT activity was inhibited. These findings agree with the increasing ROS level in human IVM oocytes.

Interestingly, the third key gene we found in sequencing results, DPYD, is a $\mathrm{NADP}^{+}$-dependent enzyme encoded by $D P Y D$ (53). It has a key role in the repair of DNA DSBs (45). Its expression in human oocytes is described here, and higher expression of DPYD was found in secondary follicles in which DNA DSBs and homologous recombination are both occurring. Importantly, we found that the genes and proteins of DPYD were activated excessively when primary follicles, secondary follicles, and MII oocytes were cultured in vitro, which suggested that the risk of DNA DSBs or chromosome segregation was increased in the in vitro environment. 
Considering that embryos from human IVM oocytes had many more tiny chromosomal defects than those from human IVO oocytes, increasing the expression of DPYD may facilitate the repair process and overcome the risk of aneuploidy. Such overexpression may explain why previous studies found that although euploidy was decreased in human IVM oocytes, $60 \%$ of them still displayed euploid chromosomes (32).

In conclusion, here we revealed a mechanism to explain why IVM oocytes can be matured in vitro but often display reduced developmental potential after fertilization (Supplementary Fig. S15). The in vitro environment can induce errors in the expression of abundant genes; however, a compensatory mechanism can help oocytes avoid failure of maturation or development. Unfortunately, the continuous increase in the demand for cellular fuel during development would probably induce organelle dysfunction and thus disrupt the balance among energy production, cell cycle progression, and nutrient metabolism. Targeting the production of a sufficient energy supply in the mitochondria is therefore a promising approach to ameliorate the poor outcome of human IVM oocytes.

\section{Methods}

\section{Collection of human oocytes}

The present study was approved by the Institutional Review Board of Peking University Third Hospital and the Third Affiliated Hospital of Guangzhou Medical University. All of the oocyte donors signed informed consent voluntarily after they were clearly informed of the content and details of this study. Two groups of women were involved in the present study. One group consisted of women who had immature oocytes after oocyte retrieval and removal of cumulus cells. These women were all undergoing ICSI cycles because of male fertility factors. In general, such immature oocytes are discarded as medical waste. Here we collected them for the analysis of specific genes. The other group consisted of women who donated their oocytes voluntarily, with no financial benefit except for the necessary nutritional and transportation fees. Three women in this group were selected for single-cell transcriptome sequencing because they had at least one IVM oocyte and one IVO oocyte. No differences were found among three donors for the main physiological indexes (Supplementary Table S7).

\section{IVM of human oocytes}

Immature human oocytes were cultured in the IVM basal medium supplemented with $0.075 \mathrm{IU} / \mathrm{mL}$ follicle-stimulating hormone, $0.075 \mathrm{IU} / \mathrm{mL}$ luteinizing hormone, $10 \mathrm{ng} / \mathrm{mL}$ epidermal growth factor, $10 \mathrm{ng} / \mathrm{mL}$ brain-derived neurotrophic factor, and $10 \mathrm{ng} / \mathrm{mL}$ insulin-like growth factor- 1 . After $24-28 \mathrm{~h}$, oocytes that expelled the first polar body were collected, transferred into Gm culture medium (LGGG-050; LifeGlobal Group, LLC), and prepared for molecular analysis.

\section{Transcriptome sequencing using single oocytes}

Single-cell transcriptome sequencing was performed by Annoroad Gene Technology Co. Ltd (Beijing, China) using six human oocytes, including three IVM and three IVO oocytes. Oocytes were rinsed in $\mathrm{Ca}^{2+} / \mathrm{Mg}^{2+}$-free PBS solution and individually placed in 0.2-mL PCR tubes containing a 5$\mu \mathrm{L}$ mixture of lysis buffer and RNase inhibitor. RNA from a single oocyte was amplified using the SMARTer Ultra Low RNA kit for Illumina sequencing (634936; Clontech Laboratories, Inc.). In brief, reverse transcriptase, buffer, oligo(dT) primers, and template switch oligo primers were added to obtain the first-strand cDNA. Then, PCR amplification solution and in situ PCR primers were added for second-strand synthesis ( $\sim 1-2 \mathrm{~kb}$ in length). The resulting cDNA was purified and recycled using AMPure XP Beads (A63882; Beckman Coulter, Inc.) and dissolved in elution buffer (EB) solution. The cDNA concentration was determined using a Qubit ${ }^{\circledR} 2.0$ Fluorometer, and the cDNA fragment length distribution was quantified using the Agilent 2100 High Sensitivity DNA Assay Kit (5067-4626; Agilent Technologies). The cDNA library was generated as follows. The quantified cDNA (20 ng) was fragmented into 200-bp lengths by ultrasonic waves using a Bioruptor ${ }^{\circledR}$ sonication system. The cDNA fragments then underwent end repair, adaptor addition, and purification using AMPure XP Beads. The products with adaptors were amplified by PCR. The different samples were marked with different index tags. PCR amplification products were electrophoresed on $2 \%$ agarose gels, and 200-300-bp DNA fragments were recovered using the CWBIO Gel Extraction Kit (CW2302A; Cwbiotech, Inc., China) and dissolved in EB. cDNA library quality was tested using an Agilent 2100 Bioanalyzer, and the effective concentration was quantified by quantitative realtime PCR. For sequencing, the effective concentration was required to be $>2 \mathrm{n} M$. The cluster was produced in c-Bot using a TruSeq PE Cluster Kit v4-cBot-HS (PE-401-4001; Illumina, Inc.), and then, 100-bp dual-sequence reads were obtained using a HiSeq 2500 machine.

\section{Read mapping, expression quantification, and clustering analysis}

All reads were mapped to the human genome (version hg19) using TopHat (version 2.0.6) with default settings. Cufflinks (version 2.0.2) was used to estimate fragments per kilobase of exon per million fragments mapped (FPKM) values (55), and genes with an FPKM value of $\geq 3$ were considered to be expressed. For subsequent analysis, the biotype classifications of genes and transcripts in the ENSEMBL annotation were used to identify noncoding genes. Hierarchical clustering was carried out using Cluster 3.0 and visualized using Java Treeview. For differential expression analysis, we aligned reads against the RefSeq human transcriptome using Bowtie software (v. 2.0.2). Expression levels were then estimated using eXpress (version 1.3.0), with genelevel effective counts and RPKM values derived from the sum of the corresponding values for all isoforms of a gene. The effective count values were then used as input for DESeq to assess differential expression, and the genes with $\log 2$-fold change $\geq 2$ and Q-value $<0.05$ were considered as differential genes. Principal component analysis was performed by a singular value decomposition of the center-scaled gene expression data matrix. All sequencing data were deposited in the Gene Expression Omnibus under accession number GSE1 10798. As for clustering analysis, the complete method was used for clustering, and the selection of distance and linkage methods was Euclidean. 


\section{Gene ontology annotation and STRING analysis}

Gene ontology (GO) analysis of DEGs was performed using DAVID and GOstat. GO terms with a $p$-value of $<0.05$ were considered to be statistically significant terms. WebGestalt was used for Kyoto Encyclopedia of Genes and Genomes (KEGG) pathway enrichment analysis. Pathway with adjusted $p$-values $<0.05$ were determined to be statistically significant pathways. Cytoscape3.1.1 was used to build the networks to show the relationships between candidate genes and GO terms or KEGG pathways. STRING analysis was performed using the STRING database. Generally, genes were grouped based on text mining, and the edges represent known protein/protein interactions. The thickness of edges represents the tightness of the interaction.

\section{Quantitative real-time PCR analysis}

As described in our previous study (14), total RNA was extracted from single oocytes using the SMARTer Ultra Low RNA kit for Illumina sequencing. Synthetic cDNA was used for quantitative real-time PCR (qPCR) with an ABI 7500 PCR machine. Gene expression was normalized to the expression of the housekeeping gene $G A P D H$. Primers were synthesized by Sangon Biotech (Shanghai) Co., Ltd. For mRNA expression identification, 16 IVM and 16 IVO human oocytes were used to extract RNA from each oocyte, respectively.

\section{Enzyme-linked immunosorbent assay}

An ELISA was performed to detect enzyme activity in culture medium from mouse oocytes $(\sim 1000$ oocytes per assay). Reagents for ELISAs were purchased from Shanghai Jijin Chemistry Technology Co. Ltd (IDH3A, CK-E94849 M; OGDH, CK-E94851 M; SDHA, CK-E94855 M; ACAT1, CK-E94860 M; HADHA, CK-E94857 M). The absorbance was measured at $450 \mathrm{~nm}$, and the concentration of the indicated enzyme was calculated. The intra- and interassay coefficients of variation were $<15 \%$ and $15 \%$, respectively, with a sensitivity of $0.38 \mathrm{ng} / \mathrm{mL}$. A standard curve was plotted using software capable of generating a four-parameter logistic curve fit, and enzyme levels were calculated with the software Curve Expert 1.3 (Curve Expert).

\section{Measurement of ATP content}

The oocyte samples were washed in PBS three times and then treated using $1 \mu M$ BODIPY FL adenosine $5^{\prime}$ triphosphate (A12410; Thermo Fisher Scientific, Inc.) for $1 \mathrm{~h}$ at room temperature in a cassette. After that, the oocyte samples were rinsed again using PBS three times. Samples were assessed under a Zeiss 710 confocal fluorescence microscope. The average fluorescence intensity per oocyte was calculated using NIH ImageJ analysis software. A total of 17 human oocytes, including 11 IVM and 6 IVO oocytes, were used here.

\section{Detection of mitochondrial content}

The oocyte samples were washed three times with PBS and then incubated with MitoTracker ${ }^{@}$ Red CMXRos dye (M7512; Thermo Fisher Scientific, Inc.). After a 45-min incubation, the oocyte samples were washed three times again using PBS and assessed under a Zeiss 710 confocal fluores- cence microscope. The average fluorescence intensity per oocyte was calculated using NIH ImageJ analysis software. A total of 14 human oocytes, including 9 IVM and 5 IVO oocytes, were used here.

\section{Measurement of intracellular glutathione}

To detect intracellular glutathione (GSH), mature oocytes were incubated with $50 \mu M$ monochlorobimane (mBCI) (M1381MP; Thermo Fisher Scientific, Inc.) at $37^{\circ} \mathrm{C}$ for $40-$ $60 \mathrm{~min}$, until a steady state was reached, before acquisition of images. mBCI fluorescence was excited at $360 \mathrm{~nm}$ and measured at $470 \mathrm{~nm}$. To compare across experiments, GSH fluorescence was expressed as a percentage of the fluorescence emitted from untreated mature oocytes imaged simultaneously. A total of 60 mouse oocytes, including 30 IVM and 30 IVO oocytes, were used here.

\section{Detection of intracellular reactive oxygen species}

Intracellular reactive oxygen species (ROS) in living oocytes were stained using the general oxidative stress indicator (CM-H2DCFDA, C6827; Thermo Fisher Scientific, Inc.). Specifically, mature oocytes were incubated with $1 \mu M$ $\mathrm{CM}-\mathrm{H} 2 \mathrm{DCFDA}$ for $30 \mathrm{~min}$ at $37^{\circ} \mathrm{C}$. After extensive washing, intracellular ROS production was assessed with a Zeiss 710 confocal fluorescence microscope at excitation and emission wavelengths of 488 and $515 \mathrm{~nm}$, respectively. The average fluorescence intensity per oocyte was calculated using NIH ImageJ analysis software. A total of 60 mouse oocytes, including 30 IVM and 30 IVO oocytes, were used here.

\section{Measurement of mitochondrial superoxide levels}

Oocyte superoxide production was quantified using MitoSOX ${ }^{\mathrm{TM}}$ red mitochondrial superoxide indicator (M36008; Thermo Fisher Scientific, Inc.), following the manufacturer's instructions. Samples were assessed under a Zeiss 710 confocal fluorescence microscope at excitation and emission wavelengths of 510 and $580 \mathrm{~nm}$, respectively. The average fluorescence intensity per oocyte was calculated using NIH ImageJ analysis software. A total of 60 mouse oocytes, including 30 IVM and 30 IVO oocytes, were used here.

\section{Measurement of NADH and oxidized mitochondrial flavoprotein $\left(F A D^{1+}\right)$ autofluorescence}

Autofluorescence intensities of NADH and FAD ${ }^{++}$in oocytes and zygotes were used to estimate mitochondrial redox potential, as described previously (9). Reduced forms of $\mathrm{NADH}$ were excited by ultraviolet light at $351 \mathrm{~nm}$, and emitted fluorescence was collected using a $435-485 \mathrm{~nm}$ bandpass filter. The oxidized form $\left(\mathrm{NAD}^{+}\right)$is nonfluorescent. In contrast to $\mathrm{NADH}$, the reduced form of the flavoprotein FADH2 is nonfluorescent. Fluorescence of the oxidized form of the flavoprotein $\left(\mathrm{FAD}^{++}\right)$was excited using the $458 \mathrm{~nm}$ line of an argon laser, and emitted fluorescence was collected through the 505-550 nm bandpass filter. Fluorescence signals were normalized to 100 on maximal oxidation of $\mathrm{FAD}^{++}$and maximal reduction of $\mathrm{NADH}$ and to 0 on maximal reduction of FADH2 and $\mathrm{NAD}^{+}$. The relative redox state of each tested 
oocyte was evaluated. A total of 60 mouse oocytes, including 30 IVM and 30 IVO oocytes, were used here.

\section{Apoptosis assay}

To label fragmented 3' DNA ends (MK500; TUNEL, CLONTECH Laboratories, Inc.) as described previously (38), blastocysts were fixed in $2 \%$ paraformaldehyde, permeabilized with $0.5 \%$ Triton X-100, and then incubated with fluorescein-labeled dUTP and terminal transferase in the dark for $1 \mathrm{~h}$ at $37^{\circ} \mathrm{C}$. Propidium iodide was used for nuclear counterstaining. Embryos were observed with a Zeiss 710 confocal fluorescence microscope.

\section{Immunocytochemistry}

Protein localization was assessed via the immunofluorescence method following methods described in our previous study (14). Briefly, oocytes were fixed in $4 \%$ paraformaldehyde and then washed three times with $0.05 M$ glycine in PBS. Cells were permeabilized with $0.2 \%$ Triton X-100 for $30 \mathrm{~min}$ and then placed in a humid chamber and blocked in $3 \% \mathrm{BSA} / \mathrm{PBS}$ for $2 \mathrm{~h}$. Incubation with a primary antibody was carried out overnight at $4^{\circ} \mathrm{C}$. After rinsing, the embryos were incubated under the same conditions with a fluorescein isothiocyanate (FITC)-conjugated secondary antibody for $2 \mathrm{~h}$. The nuclear status of embryos was evaluated by staining with $10 \mu \mathrm{g} / \mathrm{mL}$ propidium iodide for $10 \mathrm{~min}$. Finally, the samples were mounted on glass slides and examined with a Zeiss 710 confocal microscope. All of the antibodies were purchased from Abcam, Inc. (PKA, ab75991; CAMKII, ab171095; CAM, ab2860; DPYD, ab54797; $\gamma \mathrm{H} 2 \mathrm{AX}$, ab26350). A total of 120 human oocytes, including 88 IVM and 32 IVO oocytes, were used here.

\section{SiRNA injection}

siRNA injection was carried out as described previously (14). In brief, siRNA powders and negative control siRNA (ncRNA) were purchased from Santa Cruz Biotechnology, Inc. [ACAT1, sc-96390 (human) and sc-108039 (mouse); HADHA, sc-75220 (human) and sc-75221 (mouse); NNT, sc-91738 (human) and sc-150013 (mouse); DPYD, SC45326; ncRNA SC-37007]. siRNA powders were diluted in dilution buffer and then injected into the cytoplasm of mouse and human oocytes. Successful inhibition was confirmed by single-cell qPCR. Oocytes injected with negative control siRNA were used as the negative control (nc) group, and uninjected oocytes were used as the control group. After microinjection, immature oocytes were cultured in IVM medium. A total of 146 human IVM oocytes were used here.

\section{Preparation of mouse oocytes}

Collection of mouse oocytes was performed as described in our previous study. Briefly, female mice ( $8-10$ weeks old) were superovulated by intraperitoneal injection of $10 \mathrm{IU}$ of pregnant mare's serum gonadotropin (PMSG) (Ningbo Hormone Production, Ningbo, China). For IVM oocytes, the ovaries were collected and punctured repeatedly at $48 \mathrm{~h}$ after PMSG injection. The separated oocytes were collected and cultured in an incubator if GV could be observed under a microscope. For IVO oocytes, the mice were injected using an injection of $10 \mathrm{IU}$ of human chorionic gonadotropin
(Ningbo Hormone Production, Ningbo, China). The cumulus-oocyte complexes were isolated from oviduct ampulla and treated with $0.3 \%$ hyaluronidase. Oocytes with the first polar body were cultured in an incubator for further experiments.

\section{Oocyte dot blot}

Oocytes of each group were treated using $4 \mu \mathrm{L}$ of RIPA lysis buffer (CW2333S; Cwbiotech, Inc., China), and then, $2 \mu \mathrm{L}$ of lysate from each group was loaded onto a PVDF membrane (EMD Millipore Corporation). After drying at room temperature, the membrane was blocked for $1 \mathrm{~h}$ in TBST $(0.2 M \mathrm{NaCl}, 0.1 \%$ Tween-20, and $10 \mathrm{~m} M$ Tris [pH 7.4]) containing $5 \%$ nonfat dry milk. The blocked membranes were then incubated with a primary antibody (Abcam) in TBST overnight at $4^{\circ} \mathrm{C}$. After that, membranes were incubated with horseradish peroxidase-conjugated anti-rabbit immunoglobulin G (IgG; 1:1000; sc-2030; Santa Cruz Biotechnology) in TBST for $1 \mathrm{~h}$ at room temperature. After each step, the membrane was washed three times for $5 \mathrm{~min}$ with TBST, and bound antibody was then detected using an enhanced chemiluminescence detection system (Carestream $4000 \mathrm{MM}$ ) according to the manufacturer's instructions. The antibodies targeted PKA, CAMKII, CAM, and NNT (ab209565). A total of 12 human oocytes, including 6 IVM and 6 IVO oocytes, were used here.

\section{Derivation of human ES cells}

The procedure for deriving human ES cells was as described in our previous studies (63). Briefly, the inner cell mass was isolated using laser equipment (ZILOS-tk; Hamilton) and was immediately transferred onto prepared feeder cells for approximately 5-7 days, and primary ESC colonies were dissociated mechanically and cultured in human ES medium consisting of 80\% Dulbecco's modified Eagle's medium (DMEM) (A1286101; Thermo Fisher Scientific, Inc.), 20\% serum replacement (10828028; Thermo Fisher Scientific, Inc.), $1 \mathrm{mM}$ glutamine (25030081; Thermo Fisher Scientific, Inc.), $1 \%$ nonessential amino acids (11140050; Thermo Fisher Scientific, Inc.), $0.1 \mathrm{~m} M$ 2-mercaptoethanol, and $50 \mathrm{UI} / \mathrm{mL}$ penicillin and $50 \mathrm{UI} / \mathrm{mL}$ streptomycin mixture. ES cell colonies were mechanically dissociated every 45 days. All human ES cells were cultured at $37^{\circ} \mathrm{C}$ and $5 \% \mathrm{CO}_{2}$ under a humidified atmosphere.

The remaining TE cell clusters were pinched in half with a needle. One part was transferred to lysis buffer for array CGH testing, and the other was cryopreserved at $-80^{\circ} \mathrm{C}$ for examination of gene expression.

\section{Array comparative genome hybridization}

Genomic DNA was extracted from TE cells and amplified using a whole-genome amplification kit (150025; Qiagen, Inc., Germany). Array CGH manipulation using a BlueGnome platform was performed according to the instruction book and as reported previously (16). In brief, the amplified DNA samples were labeled with $\mathrm{Cy} 3$ or $\mathrm{Cy} 5$ and mixed with control human DNA. After dried and resuspended, the samples were loaded onto the BlueGnome 24Sure V3 arrays. The data were analyzed using BlueFuse software (BlueGnome). 


\section{Statistics}

The results were compared using SPSS 17.0 software. An independent-samples $t$-test was performed to compare gene expression data from the two groups. A one-way ANOVA was used when comparing data from three or more groups. A chi-squared analysis was used to compare the numbers of embryos between two groups. $p$-Values of $<0.05$ were considered to indicate significant differences. Each experiment was repeated at least three times for independent biological replicates.

\section{Study approval}

Mice used in the present study were housed and bred in the Animal Center of the Medical College of Peking University according to national legislation for animal care. Human oocytes were obtained from Peking University Third Hospital and the Third Affiliated Hospital of Guangzhou Medical University, and rabbit and nonhuman primate oocytes were obtained from the Yunnan Key Laboratory of Primate Biomedical Research, Institute of Primate Translational Medicine. All the protocols in the present studies were approved by the IRB of Peking University Third Hospital, the Third Affiliated Hospital of Guangzhou Medical University, and the Institute of Primate Translational Medicine. The collection of human oocytes strictly followed the guidelines legislated and posted by the Ministry of Health of the People's Republic of China, including the "Technical Regulation for Human Assisted Reproductive Technology" and "Ethical Guiding Principles for the Research of Human ESCs."

\section{Authors' Contributions}

H.C.Z. performed collection and IVM of human oocytes, and charged for the identification of screened genes and their encoding proteins using qPCR, dot-blot, and immunohistochemistry. Y.Z. performed bioinformatic analysis; T.J.T. performed mouse study; T.T. collected rabbit and monkey oocytes, did the qPCR experiment, and charged for bioinformatic analysis. C.Y.L. performed siRNA injection experiment; Y.L.L. performed rat oocyte collection and qPCR identification. L.C. charged for aCGH analysis; N.H. took part in critical discussion and data analysis; C.L. joined bioinformatic analysis; Y.F. and Y.Y. joined in study design, article drafting, and critical discussion and article submission. R.L. charged for the management of clinical patients, and mainly contributed to the article revision; J.Q. contributed to the conception of design and coordinated the research and article editing.

\section{Acknowledgments}

This work was supported, in part, by the National Key R\&D Program of China (2016YFC1000601), the Ministry of Science and Technology of China Grants (2014CB943203), the National Natural Science Funds for general program (81571400, 31501201, 81771580, 81570101, and 31371521), and the Beijing Nova Program (xxjh2015011).

\section{Author Disclosure Statement}

All authors declare no competing financial interests.

\section{References}

1. Ali A, Benkhalifa M, and Miron P. In-vitro maturation of oocytes: biological aspects. Reprod Biomed Online 13: 437446, 2006.

2. Boerjan ML, Baarends WM, and Ruven HJ. A cytochemical staining procedure for succinate dehydrogenase activity in pre-ovulatory mouse oocytes embedded in low gelling temperature agarose. J Histochem Cytochem 39: 135-138, 1991.

3. Canto C, Gerhart-Hines Z, Feige JN, Lagouge M, Noriega L, Milne JC, Elliott PJ, Puigserver P, and Auwerx J. AMPK regulates energy expenditure by modulating NAD+ metabolism and SIRT1 activity. Nature 458: 1056-1060, 2009.

4. Carpenter K, Pollitt RJ, and Middleton B. Human liver long-chain 3-hydroxyacyl-coenzyme A dehydrogenase is a multifunctional membrane-bound beta-oxidation enzyme of mitochondria. Biochem Biophys Res Commun 183: 443448, 1992.

5. Chakraborti T, Das S, Mondal M, Roychoudhury S, and Chakraborti S. Oxidant, mitochondria and calcium: an overview. Cell Signal 11: 77-85, 1999.

6. Chian RC, Uzelac PS, and Nargund G. In vitro maturation of human immature oocytes for fertility preservation. Fertil Steril 99: 1173-1181, 2013.

7. Dalton TP, Shertzer HG, and Puga A. Regulation of gene expression by reactive oxygen. Annu Rev Pharmacol Toxicol 39: 67-101, 1999.

8. Di Emidio G, Falone S, Vitti M, D’Alessandro AM, Vento M, Di Pietro C, Amicarelli F, and Tatone C. SIRT1 signalling protects mouse oocytes against oxidative stress and is deregulated during aging. Hum Reprod 29: 2006-2017, 2014.

9. Dumollard R, Ward Z, Carroll J, and Duchen MR. Regulation of redox metabolism in the mouse oocyte and embryo. Development 134: 455-465, 2007.

10. Dunning KR, Cashman K, Russell DL, Thompson JG, Norman RJ, and Robker RL. Beta-oxidation is essential for mouse oocyte developmental competence and early embryo development. Biol Reprod 83: 909-918, 2010.

11. Dupont J, Reverchon M, Bertoldo MJ, and Froment P. Nutritional signals and reproduction. Mol Cell Endocrinol 382: 527-537, 2014.

12. Edwards RG. Maturation in vitro of mouse, sheep, cow, pig, rhesus monkey and human ovarian oocytes. Nature 208: 349-351, 1965.

13. Fan J, Shan C, Kang HB, Elf S, Xie J, Tucker M, Gu TL, Aguiar M, Lonning S, Chen H, Mohammadi M, Britton LM, Garcia BA, Aleckovic M, Kang Y, Kaluz S, Devi N, Van Meir EG, Hitosugi T, Seo JH, Lonial S, Gaddh M, Arellano M, Khoury HJ, Khuri FR, Boggon TJ, Kang S, and Chen J. Tyr phosphorylation of PDP1 toggles recruitment between ACAT1 and SIRT3 to regulate the pyruvate dehydrogenase complex. Mol Cell 53: 534-548, 2014.

14. Fan Y, Zhao HC, Liu J, Tan T, Ding T, Li R, Zhao Y, Yan J, Sun X, Yu Y, and Qiao J. Aberrant expression of maternal Plk1 and Detn3 results in the developmental failure of human in-vivo- and in-vitro-matured oocytes. Sci Rep 5: 8192, 2015.

15. Feng R, Sang Q, Kuang Y, Sun X, Yan Z, Zhang S, Shi J, Tian G, Luchniak A, Fukuda Y, Li B, Yu M, Chen J, Xu Y, Guo L, Qu R, Wang X, Sun Z, Liu M, Shi H, Wang H, Feng Y, Shao R, Chai R, Li Q, Xing Q, Zhang R, Nogales E, Jin L, He L, Gupta ML, Jr., Cowan NJ, and Wang L. Mutations in TUBB8 and Human Oocyte Meiotic Arrest. N Engl J Med 374: 223232, 2016. 
16. Fiorentino F, Spizzichino L, Bono S, Biricik A, Kokkali G, Rienzi L, Ubaldi FM, Iammarrone E, Gordon A, and Pantos K. PGD for reciprocal and Robertsonian translocations using array comparative genomic hybridization. Hum Reprod 26: 1925-1935, 2011.

17. Fulka J, Jr., First NL, and Moor RM. Nuclear and cytoplasmic determinants involved in the regulation of mammalian oocyte maturation. Mol Hum Reprod 4: 41-49, 1998.

18. Ge J, Zhai W, Cheng B, He P, Qi B, Lu H, Zeng Y, and Chen X. Insulin induces human acyl-coenzyme A: cholesterol acyltransferase 1 gene expression via MAP kinases and CCAAT/enhancer-binding protein alpha. J Cell Biochem 114: 2188-2198, 2013.

19. Gonzalez-Serrano AF, Pirro V, Ferreira CR, Oliveri P, Eberlin LS, Heinzmann J, Lucas-Hahn A, Niemann H, and Cooks RG. Desorption electrospray ionization mass spectrometry reveals lipid metabolism of individual oocytes and embryos. PLoS One 8: e74981, 2013.

20. Guo H, Zhu P, Yan L, Li R, Hu B, Lian Y, Yan J, Ren X, Lin S, Li J, Jin X, Shi X, Liu P, Wang X, Wang W, Wei Y, Li X, Guo F, Wu X, Fan X, Yong J, Wen L, Xie SX, Tang F, and Qiao J. The DNA methylation landscape of human early embryos. Nature 511: 606-610, 2014.

21. Henderson NS. Isozymes of isocitrate dehydrogenase: subunit structure and intracellular location. J Exp Zool 158: 263-273, 1965.

22. Homa ST, Carroll J, and Swann K. The role of calcium in mammalian oocyte maturation and egg activation. Hum Reprod 8: 1274-1281, 1993.

23. Hongo S, Watanabe T, Arita S, Kanome T, Kageyama H, Shioda S, and Miyazaki A. Leptin modulates ACAT1 expression and cholesterol efflux from human macrophages. Am J Physiol Endocrinol Metab 297: E474-E482, 2009.

24. Hou X, Zhang L, Han L, Ge J, Ma R, Zhang X, Moley K, Schedl T, and Wang Q. Differing roles of pyruvate dehydrogenase kinases during mouse oocyte maturation. J Cell Sci 128: 2319-2329, 2015.

25. Hou Y, Fan W, Yan L, Li R, Lian Y, Huang J, Li J, Xu L, Tang F, Xie XS, and Qiao J. Genome analyses of single human oocytes. Cell 155: 1492-1506, 2013.

26. Huang Y, Yu Y, Gao J, Li R, Zhang C, Zhao H, Zhao Y, and Qiao J. Impaired oocyte quality induced by dehydroepiandrosterone is partially rescued by metformin treatment. PLoS One 10: e0122370, 2015.

27. Kano M, Fukao T, Yamaguchi S, Orii T, Osumi T, and Hashimoto T. Structure and expression of the human mitochondrial acetoacetyl-CoA thiolase-encoding gene. Gene 109: 285-290, 1991.

28. Kanome T, Watanabe T, Nishio K, Takahashi K, Hongo S, and Miyazaki A. Angiotensin II upregulates acyl-CoA: cholesterol acyltransferase-1 via the angiotensin II Type 1 receptor in human monocyte-macrophages. Hypertens Res 31: 1801-1810, 2008.

29. Korman SH. Inborn errors of isoleucine degradation: a review. Mol Genet Metab 89: 289-299, 2006.

30. La Rovere RM, Roest G, Bultynck G, and Parys JB. Intracellular $\mathrm{Ca} 2+$ signaling and $\mathrm{Ca} 2+$ microdomains in the control of cell survival, apoptosis and autophagy. Cell Calcium 60:74-87, 2016.

31. Lemarie A and Grimm S. Mutations in the heme b-binding residue of SDHC inhibit assembly of respiratory chain complex II in mammalian cells. Mitochondrion 9: 254-260, 2009.
32. Li Y, Feng HL, Cao YJ, Zheng GJ, Yang Y, Mullen S, Critser JK, and Chen ZJ. Confocal microscopic analysis of the spindle and chromosome configurations of human oocytes matured in vitro. Fertil Steril 85: 827-832, 2006.

33. Liu XM, Zhang YP, Ji SY, Li BT, Tian X, Li D, Tong C, and Fan HY. Mitoguardin-1 and -2 promote maturation and the developmental potential of mouse oocytes by maintaining mitochondrial dynamics and functions. Oncotarget 7: 1155-1167, 2016.

34. Luddi A, Capaldo A, Focarelli R, Gori M, Morgante G, Piomboni $\mathrm{P}$, and De Leo $\mathrm{V}$. Antioxidants reduce oxidative stress in follicular fluid of aged women undergoing IVF. Reprod Biol Endocrinol 14: 57, 2016.

35. Masri S, Patel VR, Eckel-Mahan KL, Peleg S, Forne I, Ladurner AG, Baldi P, Imhof A, and Sassone-Corsi P. Circadian acetylome reveals regulation of mitochondrial metabolic pathways. Proc Natl Acad Sci U S A 110: 3339 3344, 2013.

36. Melo AS, Kliemchen J, Junior AA, Ferriani RA, and Navarro PA. Oxidative stress and polycystic ovary syndrome: evaluation during ovarian stimulation for ICSI. Reproduction 2016. pii: REP-16-0084. [Epub ahead of print].

37. Menezo YJ, Silvestris E, Dale B, and Elder K. Oxidative stress and alterations in DNA methylation: two sides of the same coin in reproduction. Reprod Biomed Online 33: 668683, 2016.

38. Moley KH, Chi MM, Knudson CM, Korsmeyer SJ, and Mueckler MM. Hyperglycemia induces apoptosis in preimplantation embryos through cell death effector pathways. Nat Med 4: 1421-1424, 1998.

39. Nickel AG, von Hardenberg A, Hohl M, Loffler JR, Kohlhaas M, Becker J, Reil JC, Kazakov A, Bonnekoh J, Stadelmaier M, Puhl SL, Wagner M, Bogeski I, Cortassa S, Kappl R, Pasieka B, Lafontaine M, Lancaster CR, Blacker TS, Hall AR, Duchen MR, Kastner L, Lipp P, Zeller T, Muller C, Knopp A, Laufs U, Bohm M, Hoth M, and Maack C. Reversal of mitochondrial transhydrogenase causes oxidative stress in heart failure. Cell Metab 22: 472 484, 2015.

40. Oyedotun KS and Lemire BD. The quaternary structure of the Saccharomyces cerevisiae succinate dehydrogenase. Homology modeling, cofactor docking, and molecular dynamics simulation studies. J Biol Chem 279: 9424-9431, 2004.

41. Paczkowski M, Silva E, Schoolcraft WB, and Krisher RL. Comparative importance of fatty acid beta-oxidation to nuclear maturation, gene expression, and glucose metabolism in mouse, bovine, and porcine cumulus oocyte complexes. Biol Reprod 88: 111, 2013.

42. Palmer A, Gavin AC, and Nebreda AR. A link between MAP kinase and p34(cdc2)/cyclin B during oocyte maturation: p90(rsk) phosphorylates and inactivates the p34(cdc2) inhibitory kinase Myt1. EMBO J 17: 5037-5047, 1998.

43. Perkins AT, Das TM, Panzera LC, and Bickel SE. Oxidative stress in oocytes during midprophase induces premature loss of cohesion and chromosome segregation errors. Proc Natl Acad Sci U S A 113: E6823-E6830, 2016.

44. Rybalka E, Timpani CA, Cooke MB, Williams AD, and Hayes A. Defects in mitochondrial ATP synthesis in dystrophin-deficient mdx skeletal muscles may be caused by complex I insufficiency. PLoS One 9: e115763, 2014.

45. Sakata K, Someya M, Matsumoto Y, Tauchi H, Kai M, Toyota M, Takagi M, Hareyama M, and Fukushima M. Gimeracil, an inhibitor of dihydropyrimidine dehydroge- 
nase, inhibits the early step in homologous recombination. Cancer Sci 102: 1712-1716, 2011.

46. Schuh $M$ and Ellenberg J. Self-organization of MTOCs replaces centrosome function during acentrosomal spindle assembly in live mouse oocytes. Cell 130: 484-498, 2007.

47. Stricker SA. Comparative biology of calcium signaling during fertilization and egg activation in animals. Dev Biol 211: 157-176, 1999.

48. Su YQ, Sugiura K, Sun F, Pendola JK, Cox GA, Handel MA, and Schimenti JC, Eppig JJ. MARF1 regulates essential oogenic processes in mice. Science 335: 1496-1499, 2012.

49. Sugiura K, Su YQ, Diaz FJ, Pangas SA, Sharma S, Wigglesworth K, O'Brien MJ, Matzuk MM, Shimasaki S, and Eppig JJ. Oocyte-derived BMP15 and FGFs cooperate to promote glycolysis in cumulus cells. Development 134: 2593-2603, 2007.

50. Sutton-McDowall ML, Gilchrist RB, and Thompson JG. The pivotal role of glucose metabolism in determining oocyte developmental competence. Reproduction 139: 685695, 2010.

51. Sutton-McDowall ML, Purdey M, Brown HM, Abell AD, Mottershead DG, Cetica PD, Dalvit GC, Goldys EM, Gilchrist RB, Gardner DK, and Thompson JG. Redox and anti-oxidant state within cattle oocytes following in vitro maturation with bone morphogenetic protein 15 and follicle stimulating hormone. Mol Reprod Dev 82: 281-294, 2015.

52. Takahashi Y, Hashimoto S, Yamochi T, Goto H, Yamanaka M, Amo A, Matsumoto H, Inoue M, Ito K, Nakaoka Y, Suzuki N, and Morimoto Y. Dynamic changes in mitochondrial distribution in human oocytes during meiotic maturation. J Assist Reprod Genet 33: 929-938, 2016.

53. Takai S, Fernandez-Salguero P, Kimura S, Gonzalez FJ, and Yamada K. Assignment of the human dihydropyrimidine dehydrogenase gene (DPYD) to chromosome region $1 \mathrm{p} 22$ by fluorescence in situ hybridization. Genomics 24: 613-614, 1994.

54. Tang F, Barbacioru C, Wang Y, Nordman E, Lee C, Xu N, Wang X, Bodeau J, Tuch BB, Siddiqui A, Lao K, and Surani MA. mRNA-Seq whole-transcriptome analysis of a single cell. Nat Methods 6: 377-382, 2009.

55. Trapnell C, Williams BA, Pertea G, Mortazavi A, Kwan G, van Baren MJ, Salzberg SL, Wold BJ, and Pachter L. Transcript assembly and quantification by RNA-Seq reveals unannotated transcripts and isoform switching during cell differentiation. Nat Biotechnol 28: 511-515, 2010.

56. Valsangkar DS and Downs SM. Acetyl CoA carboxylase inactivation and meiotic maturation in mouse oocytes. Mol Reprod Dev 82: 679-693, 2015.

57. Van Blerkom J. Mitochondria in early mammalian development. Semin Cell Dev Biol 20: 354-364, 2009.

58. Xue Z, Huang K, Cai C, Cai L, Jiang CY, Feng Y, Liu Z, Zeng Q, Cheng L, Sun YE, Liu JY, Horvath S, and Fan G. Genetic programs in human and mouse early embryos revealed by single-cell RNA sequencing. Nature 500: 593-597, 2013.

59. Yan L, Yang M, Guo H, Yang L, Wu J, Li R, Liu P, Lian Y, Zheng X, Yan J, Huang J, Li M, Wu X, Wen L, Lao K, Li R, Qiao J, and Tang F. Single-cell RNA-Seq profiling of human preimplantation embryos and embryonic stem cells. Nat Struct Mol Biol 20: 1131-1139, 2013.

60. Yin S, Jiang X, Jiang H, Gao Q, Wang F, Fan S, Khan T, Jabeen N, Khan M, Ali A, Xu P, Pandita TK, Fan HY, Zhang $\mathrm{Y}$, and Shi Q. Histone acetyltransferase KAT8 is essential for mouse oocyte development by regulating reactive oxygen species levels. Development 144: 2165-2174, 2017.
61. Yu C, Ji SY, Sha QQ, Sun QY, and Fan HY. CRL4-DCAF1 ubiquitin E3 ligase directs protein phosphatase 2A degradation to control oocyte meiotic maturation. Nat Commun 6: 8017, 2015.

62. Yu C, Zhang YL, Pan WW, Li XM, Wang ZW, Ge ZJ, Zhou JJ, Cang Y, Tong C, Sun QY, and Fan HY. CRL4 complex regulates mammalian oocyte survival and reprogramming by activation of TET proteins. Science 342: 1518-1521, 2013.

63. Yu Y, Chang L, Zhao H, Li R, Fan Y, and Qiao J. Chromosome microduplication in somatic cells decreases the genetic stability of human reprogrammed somatic cells and results in pluripotent stem cells. Sci Rep 5: 10114, 2015.

64. Yu Y, Mai Q, Chen X, Wang L, Gao L, Zhou C, and Zhou Q. Assessment of the developmental competence of human somatic cell nuclear transfer embryos by oocyte morphology classification. Hum Reprod 24: 649-657, 2009.

65. Zhang M, Su YQ, Sugiura K, Xia G, and Eppig JJ. Granulosa cell ligand NPPC and its receptor NPR2 maintain meiotic arrest in mouse oocytes. Science 330: 366-369, 2010.

66. Zhao HC, Ding T, Ren Y, Li TJ, Li R, Fan Y, Yan J, Zhao Y, Li M, Yu Y, and Qiao J. Role of Sirt3 in mitochondrial biogenesis and developmental competence of human in vitro matured oocytes. Hum Reprod 31: 607-622, 2016.

67. Zong NC, Li H, Li H, Lam MP, Jimenez RC, Kim CS, Deng N, Kim AK, Choi JH, Zelaya I, Liem D, Meyer D, Odeberg J, Fang C, Lu HJ, Xu T, Weiss J, Duan H, Uhlen M, Yates JR, 3rd, Apweiler R, Ge J, Hermjakob H, and Ping P. Integration of cardiac proteome biology and medicine by a specialized knowledgebase. Circ Res 113: 1043-1053, 2013.

Address correspondence to: Dr. Rong $\mathrm{Li}$ Department of Obstetrics and Gynecology Peking University Third Hospital No. 49 HuaYuan North Road HaiDian District Beijing 100191 People's Republic of China

E-mail: roseli001@sina.com Dr. Yang $Y u$ Department of Obstetrics and Gynecology Peking University Third Hospital No. 49 HuaYuan North Road HaiDian District Beijing 100191 People's Republic of China E-mail: yuyang5012@hotmail.com

Dr. Yong Fan Key Laboratory for Major Obstetric Diseases of Guangdong Province

The Third Affiliated Hospital of Guangzhou Medical University

DuoBao Road 63 Guangzhou 510150

China

E-mail: fanyong0413@sina.com

Date of first submission to ARS Central, May 9, 2017; date of final revised submission, February 20, 2018; date of acceptance, February 25, 2018. 


$\begin{aligned} & \text { Abbreviations Used } \\ \text { ADCY } & =\text { adenylyl cyclase } \\ \text { ALDH } & =\text { aldehyde dehydrogenase } \\ \text { CAM } & =\text { calmodulin } \\ \text { CAMKII } & =\text { calmodulin-dependent Protein Kinase II } \\ \text { DEG } & =\text { differentially expressed gene } \\ \text { DSBs } & =\text { double-strand breaks } \\ \text { EB } & =\text { elution buffer } \\ \text { ELISA } & =\text { enzyme-linked immunosorbent assay } \\ \mathrm{ER} & =\text { endoplasmic reticulum } \\ \mathrm{ES} & =\text { embryonic stem } \\ \mathrm{FITC} & =\text { fluorescein isothiocyanate } \\ \mathrm{GO} & =\text { gene ontology } \\ \mathrm{GSH} & =\text { glutathione } \\ \mathrm{HQ} & =\text { high quality }\end{aligned}$

$\begin{aligned} \mathrm{ICSI} & =\text { intracytoplasmic sperm injection } \\ \mathrm{IVM} & =\text { in vitro } \text { maturation } \\ \mathrm{IVO} & =\text { in vivo } \\ \mathrm{KEGG} & =\text { Kyoto Encyclopedia of Genes and Genomes } \\ \mathrm{LQ} & =\text { low quality } \\ \mathrm{MAPK} & =\text { mitogen-activated protein kinase } \\ \mathrm{mBCI} & =\text { monochlorobimane } \\ \mathrm{ncRNA} & =\text { negative control siRNA } \\ \mathrm{NNT} & =\text { nicotinamide nucleotide transhydrogenase } \\ \mathrm{PCOS} & =\text { polycystic ovary syndrome } \\ \mathrm{PI} 3 \mathrm{~K} & =\text { phosphatidylinositol } 3 \text {-kinase } \\ \mathrm{PMSG} & =\text { pregnant mare's serum gonadotropin } \\ \mathrm{PRK} & =\text { protein kinase } \\ \mathrm{qPCR} & =\text { quantitative real time PCR } \\ \mathrm{ROS} & =\text { reactive oxygen species } \\ \mathrm{TE} & =\text { trophectoderm }\end{aligned}$

